\title{
Cocaine causes rapid remodeling of mesoaccumbens dopaminergic axons, synapses, and mitochondria
}

\section{Gregg Wildenberg}

The University of Chicago/Argonne National Lab

\section{Anastasia Sorokina}

University of Chicago

\section{Jessica Koranda}

The University of Chicago

\section{Alexis Monical}

The University of Chicago

\section{Chad Heer}

The University of Chicago https://orcid.org/0000-0002-4527-0405

\section{Mark Sheffield}

University of Chicago https://orcid.org/0000-0003-0969-7820

\section{Xiaoxi Zhuang}

The University of Chicago

\section{Daniel McGehee}

University of Chicago

Narayanan Kasthuri ( $\nabla$ bobbykasthuri@uchicago.edu )

University of Chicago

\section{Article}

Keywords: addiction, cocaine, drug abuse

Posted Date: January 21st, 2021

DOl: https://doi.org/10.21203/rs.3.rs-136861/v1

License: (c) (i) This work is licensed under a Creative Commons Attribution 4.0 International License. Read Full License 
Title: Cocaine causes rapid remodeling of mesoaccumbens dopaminergic axons, synapses, and mitochondria

Authors: G.A. Wildenberg ${ }^{1,2^{*}}$, A.M. Sorokina ${ }^{1,2}$, J.L. Koranda ${ }^{1}$, A. Monical ${ }^{3}$, C. Heer ${ }^{1}$, M.E. Sheffield ${ }^{1}$, X. Zhuang ${ }^{1}$, DS McGehee ${ }^{3}$, N. Kasthuri ${ }^{1,2}$

\author{
Affiliations: \\ ${ }^{1}$ Department of Neurobiology, University of Chicago, Chicago, IL, USA. \\ ${ }^{2}$ Argonne National Laboratory, Lemont, IL, USA. \\ ${ }^{3}$ Department of Anesthesia \& Critical Care, University of Chicago, Chicago, IL, USA. \\ *Correspondence to: bobbykasthuri@uchicago.edu and gwildenberg@uchicago.edu
}

\title{
Summary
}

Detailing the ways drugs of abuse physically alter dopaminergic circuits would provide new mechanisms for explaining addictive behaviors, future targets for therapeutic intervention, and insights into the nature of synaptic plasticity. We combine recent advances in genetic labeling with large volume serial electron microscopy to detail how normal dopaminergic (DA) axons interact with putative targets in the Nucleus Accumbens (NAc) and how those interactions change in mice briefly exposed to cocaine. We find that while most DA axonal boutons are devoid of obvious signs of synapses (i.e. synaptic vesicles or synaptic densities), many DA boutons physically interdigitate with dendrites or excitatory and inhibitory axons. A brief exposure to cocaine results in large-scale remodeling: extensive DA axonal branching and frequent occurrences of axonal blind-ended "bulbs", filled with mitochondria and reminiscent of axonal retraction in the developing and damaged brain. The number of physical interdigitations and vesicle filled boutons in DA axons scales linearly with the length of axon in both controls and cocaine exposed animals and the size or the type of interaction (i.e. axo-axonic or axo-dendritic) do not change. Finally, we find in cocaine exposed animals, mitochondrial lengths are increased $\sim 2.5$ times relative to control. Mitochondrial elongation is cell type specific: primarily in DA neurons and downstream spiny dendrites, and localized to DA axons and not DA soma or dendrites. We show for the first time the effects of cocaine on remodeling of dopamine axon morphology and mitochondria and reveal new details on how dopamine neurons physically associate with downstream targets.

\section{Introduction}

It is well established that alterations in the mesoaccumbens dopamine pathway, from ventral tegmental area (VTA) to nucleus accumbens (NAc), is central to the development of addictive behaviors ${ }^{1}$. The primary effects of cocaine, a strong, stimulant, is likely caused by physical binding to the dopamine transporter (DAT) on DA 
neurons, preventing reuptake of extracellular dopamine, and resulting in prolonged dopamine-mediated modulation of target neurons ${ }^{2}$. This leads to persistent functional and structural plasticity in neurons of the mesoaccumbens pathway. Cocaine-mediated changes in Long-term potentiation (LTP) and Long-term depression (LTD) have been reported in pathways upstream of DA neurons between excitatory inputs and dopamine dendrites in the VTA ${ }^{3,4}$ and downstream of DA neurons at synaptic sites of NAc-residing Medium spiny neurons (MSNs) $)^{5,6}$. Structurally, cocaine has been reported to increase the spine density in DA dendrites in the $\mathrm{VTA}^{7}$, and decrease dendritic branching and spine density of NAc MSNs ${ }^{8,9}$.

However, there exists little information on how cocaine affects DA axons and their connections. Part of the reason for this gap is the difficulty in characterizing connectivity in general and the connectivity of dopamine axons specifically,as there is not a 'pathognomonic' anatomical signature of DA axons or their 'connections' with targets ${ }^{10,11}$. Previous EM studies of DA axons, primarily limited to immuno-labeling approaches of single 2D EM sections, report that $\mathrm{TH}+$ (i.e. DA) axons are in close proximity to NAc dendrites often receiving a second spine synapse from a VGLUT+ axon terminal ${ }^{12-14}$. These putative points of contact between DA axons and NAc neurites showed weak expression of synaptic proteins (e.g. Gephyrin, PSD-95, AMPAR) and thus may represent points of molecularly organized physical interactions ${ }^{15}$. Finally, experiments using fluorescence labeling has suggested that DA axons form varicosities in close proximity to dendritic spines and the number of these dendritic-neighboring varicosities increase after acute cocaine treatment ${ }^{16}$. While suggestive, the lack of anatomical gold-standard markers classically used to define synaptic contacts ${ }^{17}$ makes it difficult to tell whether a neurites proximity to a DA axon is conclusive evidence for being a dopamine target. Thus, results on how cocaine modifies DA axon structure and potential synapses remains elusive.

We address this gap by combining recent advances in genetic labeling techniques for EM along with large volume, serial EM (i.e. "connectomics") ${ }^{18}$ to study how cocaine alters the structure of dopamine axons. Using the genetically encoded pea peroxidase gene, APEX $2^{19,20}$, we labeled VTA dopamine neurons in 2 cocaine sensitized and 3 control mice in serial EM datasets and reconstructed dopamine neurons in the VTA and their axons in the NAc ( $\sim 0.5 \mathrm{~mm} \times 0.5 \mathrm{~mm} \times 0.2 \mathrm{~mm}$ volumes). We show that cocaine exposure causes widespread remodeling of DA axons with extensive branching, the formation of 'bulbs', reminiscent of retraction bulbs, and widespread changes in mitochondria in DA axons and their putative targets. Our findings give new insight into DA axon biology and help fill in a major gap in describing how cocaine impacts the mesoaccumbens dopamine circuitry.

\section{Results}

To selectively label mesoaccumbens dopamine neurons, we bilaterally injected Adenoassociated virus (AAV) encoding either a CRE-dependent cytosolic or mitochondrial targeted pea peroxidase, Apex2, into the Ventral Tegmental Area (VTA) of 5 mice 
expressing CRE recombinase from the promoter of the dopamine transporter gene (DAT-CRE) (Fig. 1a) ${ }^{21}$. This strategy effectively labeled VTA-residing DA neurons that project axons to the Nucleus Accumbens (NAc). Four weeks following AAV expression, mice were perfused and brain slices were treated with 3,3'-Diaminobenzidine (DAB) to precipitate and convert Apex2 into a polymer with binding affinity to osmium tetroxide ${ }^{19,20}$ (Fig. 1b). Brain sections with Apex2-precipitate in appropriate regions (i.e. VTA and NAc) were cut into smaller pieces (VTA and NAc, Fig. 1b, right green circles) and further processed for serial $\mathrm{EM}^{22}$ (see Methods). Single 2D EM sections confirmed that both cytosolic and mitochondrial targeted Apex2 unambiguously labeled DA neurons at their somata (Fig. 1c, left panels, red arrow), dendrites (Fig. 1c, middle panels, cyan arrow) and thin axonal processes several millimeters away from the injection site (Fig. 1c, right panel, yellow arrow and arrowhead). We found no evidence of labeled somata in any other brain region, including the NAc, suggesting that the viral strategy targeted genetically specified DA axons in an anterograde manner. In 4 animals, AAV cytosolic Apex2 injections in DAT-CRE mice were followed four weeks later by an established cocaine sensitization and associative conditioning protocol that has been previously shown to induce morphological changes in the the mesoaccumbens dopamine pathway ${ }^{23-25}$. Briefly, mice were given one daily intraperitoneal (IP) injection of either cocaine (2 mice; $10 \mathrm{mg} / \mathrm{kg})$ or equivalent volume of saline (2 mice) every other day for a total of 4 injections (Fig. 1d, left panel). Immediately following each cocaine injection, mice were place in a novel environment where locomotor activity was monitored. Figure $1 \mathrm{e}$ shows an example of the increase in animal movements between cocaine and saline treated groups after the first day of treatment (see Supplementary Figure 1 for full behavioral data). After the final injection, mice went through a four-day abstinence period before being sacrificed and their brains processed for Apex2 staining and serial EM. A four-day abstinence period was chosen to reduce any acute effects of cocaine and ensure our results reflected long term changes caused by cocaine sensitization ${ }^{26-31}$.

We first described ultra-structural features of the putative DA contacts with target neurons (i.e. what, if any, types of synapses did they make?). We focused on 30 Apex2 labeled boutons from DA axons in control mice $(n=2)$ and 20 from the cocaine treated mice $(n=2)$ and for each bouton, examined every neuron in membrane to membrane apposition for signs of synaptic specialization (i.e. pre-synaptic vesicles, post-synaptic densities). In order to ensure that we did not miss relevant ultrastructural details, we also examined DA boutons in control animals where we injected an AAV containing a CRE-dependent mitochondrial targeted Apex2 into a DAT-CRE mouse. By targeting Apex2 to mitochondria, we were able to identify DA axons in the EM while also insuring complete visualizing of their internal contents (i.e. synaptic vesicles). Broadly, most DA boutons $(65 \%$ or $67 / 102)$ contained a few synaptic vesicles, but only in few $\sim 2 \%(2 / 102)$ could we see any sign of post-synaptic density staining in any neurite in contact with the bouton, although PSDs were easy to identify in neighboring unlabeled excitatory axonal synapses. An interesting feature of DA axons in both Apex-cyto and Apex-Mito datasets was that $20 \%(21 / 102)$ of boutons contained membrane invaginations, as physical points of contact with either unlabeled axons (control: $83 \%, n=49 / 59$ contact points scored across 19 axons; cocaine: $77 \%, n=56 / 73$ contact points scored across 20 
axons) or unlabeled dendrites (control: $17 \%, n=10 / 59$ contact points scored across 19 axons; cocaine: $23 \%, n=17 / 73$ contact points scored across 19 axons) (Figure 2a-b). Of the unlabeled axons, $43 \%$ (6/13) made additional synapses on dendritic spines in the volume, suggesting they were excitatory and the rest, $57 \%$, made synapses on dendritic shafts with little sign of a PSD, suggesting they were inhibitory. All dendrites contacted were $100 \%$ spiny. These invaginations were the main signs of physical interactions between DA axons and putative targets and we saw no evidence of innervation of dendritic spines, (i.e. a DA bouton proximate to a spine (or shaft) with a clear postsynaptic density).

We next asked how DA neurons and their interactions were influenced by cocaine with a specific focus on anatomical rearrangements of DA axons. After sensitizing mice to cocaine (see Fig. 1, Supplementary Fig. 1, and Methods), we first analyzed the trajectories of axons in $20 \mathrm{~nm}(\mathrm{x}, \mathrm{y})$ resolution EM volumes from NAc datasets of 2 control (+saline) and 2 cocaine treated (+cocaine) mice. We traced 85 Apex2+ DA axons (total length of 5192.8 microns) and what became immediately obvious from the reconstructions was that DA axons branched far more than controls after exposure to cocaine (+ cocaine, red axons, +saline, blue axons; Figure 3a). Across labeled DA axons, there was an average 4.9-fold increase in branch number for similar axon lengths with exposure to cocaine (Fig. 3b; branch number/axon: +saline, 0.61 mean, $\mathrm{n}=$ 44 axons; +cocaine, 2.96 mean, $n=41$ axons. $P=3.96 e-8)$. This increased branching was accompanied by an increased number of interactions with post-synaptic targets as described above, but the number of contacts per length was the same between cocaine exposed and control DA axons. (Figure 3c; number of contacts points/axon: +saline, 12.5 mean, $r^{2}=0.94, n=125$ contact points across 10 axons; +cocaine, 15.8 mean, $r^{2}=$ $0.54, n=142$ contact points across 9 axons. $P=0.90$ ). Indeed, control axons showed fairly regular and uniform distributions of contacts along the axon (Fig. 3a), whereas in cocaine exposed and highly branched DA axons, there were large regions of axon that were devoid of any contacts (Fig. 3a). Finally, we did not see an obvious difference in the distribution of invaginations that were axo-axonic versus axo-dendritic or contained vesicles (Supplementary Fig. 2: control: 83\% (49/59) axo-axonic, 17\% (10/59) axodendritic; cocaine: $77 \%$ (56/73) axo-axonic, 23\% (17/73) axo-dendritic), suggesting that while there were large changes in axonal structure and the numbers of contact points, there were minimal changes in how DA axons interacted with post-synaptic targets.

The second obvious feature of DA axons exposed to cocaine was the occurrence of large swellings or bulbs in the axon (Fig. 4). The swellings were often $>600$ nanometers in diameter and at times reaching the size of neuronal somata (Supplementary Figure 3 ), significantly larger than those associated with interdigitations (102 nanometers mean, $\mathrm{n}=30$ contact points.) described above. These 'bulbs' were common in the cocaine exposed animals $\sim 56 \%$ ( $17 / 30$ axons) but we did not see a single example in DA axons from control animals, suggesting that Apex2 expression alone does not cause these swellings (Figure 4a; swellings/axon: + saline, 0.00 mean, $n=29$ axons; + cocaine, 1.00 mean, $n=30$ axons. $P=8.0 \mathrm{e}-6)$. Figure $4 \mathrm{~b}$ shows reconstructions of two of these axons with swellings (large spheres with asterisk). DA axons were found to have either a large swelling along the axon (bottom reconstruction), often surrounded by 
medium sized swellings (green spheres), or contained terminal bulbs (top reconstruction, asterisk) reminiscent of axon retraction bulbs observed in developing neuromuscular junctions and developing brains ${ }^{32-36}$. Interestingly, we did not observe such any swellings in any NAc dendrite or afferent axons (i.e. excitatory axons that make chemical synapses) coming from cortical and subcortical areas ${ }^{37}$. Additionally, we did not observe any swellings in VTA DA dendrites despite also being sites of dopamine release (data not shown). We next re-imaged EM volumes around different swellings at a higher resolution $(\sim 6 \mathrm{~nm} x, y)$ to better resolve the contents of these structures. Figure 4c shows two examples (highlighted in red), one containing Apex2 (Apex+) and one that did not (Apex-). The Apex- axon was verified to structurally resemble DA axons that likely did not get infected with the Apex2 AAV (i.e. contained varicosities, did not make chemical synapses, nor dendritic spines). Both swellings were completely filled with elongated mitochondria (highlighted in blue) and surrounded by otherwise normal neuropil (example of dendrites and myelinated axons highlighted in green). When these bulbs were reconstructed into 3D renderings, we found the mitochondria to be extremely elongated and twisted when packed into the swelling (Figure 4d) and that nearly all bulbs examined were packed this way.

The tortuous and elongated nature of the mitochondria in these bulbs made us curious about possible mitochondrial changes in other cell types in the same tissue. One advantage of large volume EM datasets is that, since all cells and intracellular organelles like mitochondria are also labeled, we could ask whether cocaine altered mitochondrial length in other parts of DA neurons as well as other neurons in the NAc. We quantified mitochondrial lengths at five locations: (Figure 5a): in the NAc, we measured mitochondria length in: (a) Apex2+ DA axons, (b) axons that make chemical synapses (i.e. GLUT2 afferents) (CS axons), and (c) MSN dendrites and in the VTA, (d) Apex2+ DA somata and (e) Apex2+ DA dendrites. Consistent with the increased mitochondria lengths found in large DA axon swellings, Apex+ DA axons in the NAc had longer mitochondria throughout in the cocaine treated mouse compared to the saline control (Fig 5b top; mitochondria length: +saline, 0.36 mean, $\mathrm{n}=162$ mitochondria across 42 axons; +cocaine, 0.79 mean, $\mathrm{n}=126$ mitochondria across 35 axons. $\mathrm{P}=$ 7.25e-25). Cocaine also resulted in increased mitochondrial length in NAc MSN dendrites, the putative targets of DA axons (Fig $5 \mathrm{~b}$ middle; mitochondria length/dendrite diameter: +saline, 1.39 mean, $\mathrm{n}=132$ mitochondria across 50 dendrites; +cocaine, 3.00 mean, $n=260$ mitochondria across 41 dendrites. $P=1.14 \mathrm{e}-6$ ). However, increased mitochondria length appeared to be specific to DA axons and MSN dendrites, as we observed no difference in mitochondrial length in Apex- NAc CS axons between cocaine and saline treated mice (Fig 5b bottom; mitochondria length: +saline, 0.70 mean, $n=$ 104 mitochondria across 30 axons; +cocaine, 0.73 mean, $\mathrm{n}=164$ mitochondria across 57 axons. $P=0.64)$. We visually summarized these results through $3 \mathrm{D}$ reconstructions shown in Supplementary Figure 4. Surprisingly, we didn't not observe any differences in mitochondrial length in VTA Apex+ DA somata or dendrites as compared to controls (Fig. 5c top; mitochondrial length in Apex+ DA Somata: +saline, 2.46 mean, $\mathrm{n}=70$ mitochondria across 4 somata; +cocaine, 2.78 mean, $n=141$ mitochondria across 5 somata. $\mathrm{P}=0.68$; Fig. $5 \mathrm{c}$ bottom; mitochondrial length/dendrite diameter $(\mathrm{nm})$ in Apex+ DA dendrites: +saline, 1.74 mean, $n=37$ mitochondria across 4 dendrites; +cocaine, 
1.85 mean, $n=53$ mitochondria across 10 dendrites. $P=0.57)$. Lastly, we confirmed that cocaine did not increase the number of mitochondria in DA axons (Supplementary Figure 5; number of mitochondria: +saline, 5.33 mean, $\mathrm{n}=96$ mitochondria across 18 axons; + cocaine, 5.35 mean, $n=107$ mitochondria across 20 axons. $P=0.72$ ). Taken together, these results indicate that cocaine sensitization increases mitochondrial length in the mesolimbic pathway in a cell-type specific manner (i.e. in DA axons and not in CS axons), but also in sub-cellular specific manner (i.e. in DA axons and not DA somata or DA dendrites).

\section{Discussion}

We used genetic labeling of DA axons for large volume serial electron microscopy to discover several novel features of axonal ultrastructure, along with potentially important changes to these DA axons following exposure of the animal to cocaine. First, we provide new insights into the physical interactions between DA axons and other neurons. How neuromodulatory neurons interact with downstream targets has long been unclear. Previous attempts at characterizing the ultra-structure (e.g. with EM immuno-labeling), were often limited to individual 2D EM sections but lacked definitive hallmarks of synaptic connections typically seen in conventional asymetric and symmetric synapses ${ }^{12-14}$. Using Apex2 labeling and large volume automated serial EM, we demonstrate that the majority of DA axons indeed lack obvious signs of synapses at most boutons but instead physically interdigitated with the membranes of other axons and dendrites. As previous reports suggest that only $30 \%$ of DA axon varicosities contain the molecular machinery for active dopamine release ${ }^{10}$, DA axons may shuttle dopamine vesicles to different varicosity sites. Given that DA neurons do not make chemical synapses but rather thought to release dopamine into the nearby extracellular space (i.e. volume transmission) ${ }^{11}$, it is interesting to find such close and direct physical interactions with other neurons. Future studies that correlate contact sites with DA activity of individual boutons would clarify the relationship between the physical interactions we observe with the concept of volume transmission.

Second, we expand our current understanding of the structural changes following cocaine exposure in the brain. While previous reports have focused on changes in DA spine density in the VTA and MSN spine/synapse density in the NAc, our results fill in an important gap to our understanding of how cocaine alters the structure of the mesoaccumbens circuit on the axonal side. Our data are consistent with active remodeling of dopamine axons: retraction bulbs on axon terminals in the process of pruning (i.e. removing connections) and increased branching (i.e. formation of new connections). These changes occurred after just 4 days of exposure and 4 days of withdrawal, suggesting a rapid pace of these large-scale anatomical changes. Follow-up studies that examine whether these alterations persist at longer time points or even in models of addiction (e.g. self-administration) would shed further light on how these rearrangements correlate with long term behavioral changes. Finally, our data are consistent with the idea that the major structural change following exposure to cocaine 
is an increase in the number of interactions between DA axons and targets as opposed to changes in the size or composition of individual connections.

Changes in DA axons in response to cocaine bare striking similarities to remodeling events observed during development and traumatic brain injury (TBI). Axonal bulbs in development and $\mathrm{TB} \mathrm{I}^{38}$ have been distinguished from each other as either axon retraction or axon degeneration ${ }^{39}$ (i.e. "Wallerian degeneration"), respectively ${ }^{33}$. Because we do not observe axon fragmentation, a hallmark of axon degeneration, but rather large bulbs connected to otherwise intact axons, the effects of cocaine on dopamine axons resemble more closely those seen during development with some notable differences. Axon retraction bulbs during development are often surrounded by glia to presumably remove contents of the pruned axon terminal ${ }^{33}$. However, we do not observe any glia on or near dopamine axon bulbs during this remodeling process and find no evidence of Apex2-labeled cytoplasm inside other cells, particularly glia, either in cytoplasm or in membrane filled vesicles, suggesting that bulb formation in this system seems physically unrelated to the actions of glial digestion of dead or damaged neuronal tissue ${ }^{40,41}$. Furthermore, axon retraction bulbs that form during development are not packed full with mitochondria in the manner cocaine-induced swellings are ${ }^{42}$ (see Fig. 4). This could be due to differences in the biology of cocaine-induced and developmental axon bulbs, or the timing in which we sacrificed the mouse after cocaine sensitization. Another important distinction is that some of the large swellings in dopamine axons were not terminal bulbs like those seen in neuromuscular junctions. Because dopamine neurons are neuromodulatory neurons characterized by having varicosities along their axon lengths ${ }^{10}$, we suspect bulbs in dopamine axons will form in both varicosities and terminal points. Finally, we observe bulbs larger than the surrounding soma, often much larger than axonal retraction bulbs seen during development (see Supplementary Fig. 3).

Our results on changes in mitochondrial length provide a potential cellular substrate for how cocaine alters brain energy homeostasis, including changes in oxidative stress, cellular respiration, and enrichment of mitochondrial-related transcripts in NAc brain slice preps ${ }^{43-47}$. Another possibility is that mitochondrial elongation is the result of hyper activity of the mesolimbic circuit, as demonstrated by the increased movement of mice exposed to cocaine (Supplementary Fig. 1). Changes in mitochondrial cytochrome oxidase staining with activity are well known (i.e. cytochrome oxidase and ocular dominance columns in cortex $)^{48,49}$ and the cell-type specific nature of our results (i.e. DA axons show elongated mitochondria but excitatory, putative glutamate, axons, immediately proximate do not) suggest that mitochondrial change is perhaps related to increased DA activity specifically. The increase in mitochondrial length in NAc dendrites is then because they are the targets of that DA activity. Finally, our observation that mitochondrial changes are primarily seen in DA axons and not DA somata or dendrites could suggest either that cocaine acts directly on DA axons or that the major influence of cocaine is on axonal activity. Consistent with this interpretation are data that show higher DAT expression in DA axons which likely results in axons being the major target for cocaine ${ }^{50}$. Finally, another plausible hypothesis is that changes in mitochondria in DA axons are the result of increased metabolism due to cocaine-mediated dopamine 
depletion and efforts by DA neurons to increase dopamine synthesis. Future experiments in which neuronal activity is tightly controlled, perhaps with optical stimulation ${ }^{51,52}$, and mitochondrial length is analyzed in differentially activated neurons could provide quantitative descriptions of activity and mitochondrial morphology. 


\section{References}

1 Berke, J. D. \& Hyman, S. E. Addiction, dopamine, and the molecular mechanisms of memory. Neuron 25, 515-532, doi:10.1016/s08966273(00)81056-9 (2000).

2 Ritz, M. C., Lamb, R. J., Goldberg, S. R. \& Kuhar, M. J. Cocaine receptors on dopamine transporters are related to self-administration of cocaine. Science 237, 1219-1223, doi:10.1126/science.2820058 (1987).

3 Ungless, M. A., Whistler, J. L., Malenka, R. C. \& Bonci, A. Single cocaine exposure in vivo induces long-term potentiation in dopamine neurons. Nature 411, 583-587, doi:10.1038/35079077 (2001).

4 Saal, D., Dong, Y., Bonci, A. \& Malenka, R. C. Drugs of abuse and stress trigger a common synaptic adaptation in dopamine neurons. Neuron $37,577-582$, doi:10.1016/s0896-6273(03)00021-7 (2003).

5 Huang, Y. H. et al. In vivo cocaine experience generates silent synapses. Neuron 63, 40-47, doi:10.1016/j.neuron.2009.06.007 (2009).

6 Thomas, M. J., Beurrier, C., Bonci, A. \& Malenka, R. C. Long-term depression in the nucleus accumbens: a neural correlate of behavioral sensitization to cocaine. Nat Neurosci 4, 1217-1223, doi:10.1038/nn757 (2001).

7 Sarti, F., Borgland, S. L., Kharazia, V. N. \& Bonci, A. Acute cocaine exposure alters spine density and long-term potentiation in the ventral tegmental area. Eur J Neurosci 26, 749-756, doi:10.1111/j.1460-9568.2007.05689.x (2007).

8 Barrientos, C. et al. Cocaine-Induced Structural Plasticity in Input Regions to Distinct Cell Types in Nucleus Accumbens. Biol Psychiatry 84, 893-904, doi:10.1016/j.biopsych.2018.04.019 (2018).

9 Alcantara, A. A. et al. Cocaine- and morphine-induced synaptic plasticity in the nucleus accumbens. Synapse 65, 309-320, doi:10.1002/syn.20849 (2011).

10 Liu, C., Kershberg, L., Wang, J., Schneeberger, S. \& Kaeser, P. S. Dopamine Secretion Is Mediated by Sparse Active Zone-like Release Sites. Cell 172, 706718.e715, doi:10.1016/j.cell.2018.01.008 (2018).

11 Rice, M. E. \& Cragg, S. J. Dopamine spillover after quantal release: rethinking dopamine transmission in the nigrostriatal pathway. Brain Res Rev 58, 303-313, doi:10.1016/j.brainresrev.2008.02.004 (2008).

12 Moss, J. \& Bolam, J. P. A dopaminergic axon lattice in the striatum and its relationship with cortical and thalamic terminals. J Neurosci 28, 11221-11230, doi:10.1523/JNEUROSCI.2780-08.2008 (2008).

13 Bérubé-Carrière, N. et al. Ultrastructural characterization of the mesostriatal dopamine innervation in mice, including two mouse lines of conditional VGLUT2 knockout in dopamine neurons. Eur J Neurosci 35, 527-538, doi:10.1111/j.14609568.2012.07992.x (2012).

14 Omelchenko, N. \& Sesack, S. R. Ultrastructural analysis of local collaterals of rat ventral tegmental area neurons: GABA phenotype and synapses onto dopamine and GABA cells. Synapse 63, 895-906, doi:10.1002/syn.20668 (2009).

15 Uchigashima, M., Ohtsuka, T., Kobayashi, K. \& Watanabe, M. Dopamine synapse is a neuroligin-2-mediated contact between dopaminergic presynaptic 
and GABAergic postsynaptic structures. Proc Natl Acad Sci U S A 113, 42064211, doi:10.1073/pnas.1514074113 (2016).

16 Dos Santos, M. et al. Cocaine increases dopaminergic connectivity in the nucleus accumbens. Brain Struct Funct 223, 913-923, doi:10.1007/s00429-0171532-x (2018).

17 Harris, K. M. \& Weinberg, R. J. Ultrastructure of synapses in the mammalian brain. Cold Spring Harb Perspect Biol 4, doi:10.1101/cshperspect.a005587 (2012).

18 Kasthuri, N. et al. Saturated Reconstruction of a Volume of Neocortex. Cell 162 , 648-661, doi:10.1016/j.cell.2015.06.054 (2015).

19 Martell, J. D., Deerinck, T. J., Lam, S. S., Ellisman, M. H. \& Ting, A. Y. Electron microscopy using the genetically encoded APEX2 tag in cultured mammalian cells. Nat Protoc 12, 1792-1816, doi:10.1038/nprot.2017.065 (2017).

20 Joesch, M. et al. Reconstruction of genetically identified neurons imaged by serial-section electron microscopy. Elife 5, doi:10.7554/eLife.15015 (2016).

21 Backman, C. M. et al. Characterization of a mouse strain expressing Cre recombinase from the $3^{\prime}$ untranslated region of the dopamine transporter locus. Genesis 44, 383-390, doi:10.1002/dvg.20228 (2006).

22 Hua, Y., Laserstein, P. \& Helmstaedter, M. Large-volume en-bloc staining for electron microscopy-based connectomics. Nat Commun 6, 7923, doi:10.1038/ncomms8923 (2015).

23 Beeler, J. A., Cao, Z. F., Kheirbek, M. A. \& Zhuang, X. Loss of cocaine locomotor response in Pitx3-deficient mice lacking a nigrostriatal pathway. Neuropsychopharmacology 34, 1149-1161, doi:10.1038/npp.2008.117 (2009).

24 Singer, B. F. et al. Amphetamine-induced changes in dendritic morphology in rat forebrain correspond to associative drug conditioning rather than nonassociative drug sensitization. Biol Psychiatry 65, 835-840, doi:10.1016/j.biopsych.2008.12.020 (2009).

$25 \mathrm{Li}, \mathrm{Y} .$, Acerbo, M. J. \& Robinson, T. E. The induction of behavioural sensitization is associated with cocaine-induced structural plasticity in the core (but not shell) of the nucleus accumbens. Eur J Neurosci 20, 1647-1654, doi:10.1111/j.14609568.2004.03612.x (2004).

26 Parsons, L. H., Smith, A. D. \& Justice, J. B. Basal extracellular dopamine is decreased in the rat nucleus accumbens during abstinence from chronic cocaine. Synapse 9, 60-65, doi:10.1002/syn.890090109 (1991).

27 Grimm, J. W., Hope, B. T., Wise, R. A. \& Shaham, Y. Neuroadaptation. Incubation of cocaine craving after withdrawal. Nature 412, 141-142, doi:10.1038/35084134 (2001).

28 Hollander, J. A. \& Carelli, R. M. Cocaine-associated stimuli increase cocaine seeking and activate accumbens core neurons after abstinence. J Neurosci 27 , 3535-3539, doi:10.1523/JNEUROSCI.3667-06.2007 (2007).

29 Eipper-Mains, J. E. et al. Effects of cocaine and withdrawal on the mouse nucleus accumbens transcriptome. Genes Brain Behav 12, 21-33, doi:10.1111/j.1601-183X.2012.00873.x (2013).

30 Nestler, E. J. Molecular basis of long-term plasticity underlying addiction. Nat Rev Neurosci 2, 119-128, doi:10.1038/35053570 (2001). 
31 Benuck, M., Lajtha, A. \& Reith, M. E. Pharmacokinetics of systemically administered cocaine and locomotor stimulation in mice. J Pharmacol Exp Ther 243, 144-149 (1987).

32 Balice-Gordon, R. J., Chua, C. K., Nelson, C. C. \& Lichtman, J. W. Gradual loss of synaptic cartels precedes axon withdrawal at developing neuromuscular junctions. Neuron 11, 801-815, doi:10.1016/0896-6273(93)90110-d (1993).

33 Bishop, D. L., Misgeld, T., Walsh, M. K., Gan, W. B. \& Lichtman, J. W. Axon branch removal at developing synapses by axosome shedding. Neuron 44, 651661, doi:10.1016/j.neuron.2004.10.026 (2004).

34 Bixby, J. L. Ultrastructural observations on synapse elimination in neonatal rabbit skeletal muscle. J Neurocytol 10, 81-100, doi:10.1007/BF01181746 (1981).

35 Korneliussen, H. \& Jansen, J. K. Morphological aspects of the elimination of polyneuronal innervation of skeletal muscle fibres in newborn rats. $J$ Neurocytol 5, 591-604, doi:10.1007/BF01175572 (1976).

36 Riley, D. A. Ultrastructural evidence for axon retraction during the spontaneous elimination of polyneuronal innervation of the rat soleus muscle. J Neurocytol 10, 425-440, doi:10.1007/BF01262414 (1981).

37 Pennartz, C. M., Groenewegen, H. J. \& Lopes da Silva, F. H. The nucleus accumbens as a complex of functionally distinct neuronal ensembles: an integration of behavioural, electrophysiological and anatomical data. Prog Neurobio/ 42, 719-761, doi:10.1016/0301-0082(94)90025-6 (1994).

38 Johnson, V. E., Stewart, W. \& Smith, D. H. Axonal pathology in traumatic brain injury. Exp Neurol 246, 35-43, doi:10.1016/j.expneurol.2012.01.013 (2013).

39 Rosenthal, J. L. \& Taraskevich, P. S. Reduction of multiaxonal innervation at the neuromuscular junction of the rat during development. J Physio/ 270, 299-310, doi:10.1113/jphysiol.1977.sp011953 (1977).

40 Canty, A. J. et al. In vivo imaging of injured cortical axons reveals a rapid onset form of Wallerian degeneration. BMC Biol 18, 170, doi:10.1186/s12915-02000869-2 (2020).

41 Yanuck, S. F. Microglial Phagocytosis of Neurons: Diminishing Neuronal Loss in Traumatic, Infectious, Inflammatory, and Autoimmune CNS Disorders. Front Psychiatry 10, 712, doi:10.3389/fpsyt.2019.00712 (2019).

42 Ertürk, A., Hellal, F., Enes, J. \& Bradke, F. Disorganized microtubules underlie the formation of retraction bulbs and the failure of axonal regeneration. $J$ Neurosci 27, 9169-9180, doi:10.1523/JNEUROSCI.0612-07.2007 (2007).

43 Dietrich, J. B. et al. Acute or repeated cocaine administration generates reactive oxygen species and induces antioxidant enzyme activity in dopaminergic rat brain structures. Neuropharmacology 48, 965-974, doi:10.1016/j.neuropharm.2005.01.018 (2005).

44 Kalivas, P. W. The glutamate homeostasis hypothesis of addiction. Nat Rev Neurosci 10, 561-572, doi:10.1038/nrn2515 (2009).

45 Volkow, N. D. et al. Changes in brain glucose metabolism in cocaine dependence and withdrawal. Am J Psychiatry 148, 621-626, doi:10.1176/ajp.148.5.621 (1991). 
46 Lehrmann, E. et al. Transcriptional profiling in the human prefrontal cortex: evidence for two activational states associated with cocaine abuse. Pharmacogenomics J 3, 27-40, doi:10.1038/sj.tpj.6500146 (2003).

$47 \quad$ Feng, J. et al. Chronic cocaine-regulated epigenomic changes in mouse nucleus accumbens. Genome Biol 15, R65, doi:10.1186/gb-2014-15-4-r65 (2014).

48 Horton, J. C. \& Hedley-Whyte, E. T. Mapping of cytochrome oxidase patches and ocular dominance columns in human visual cortex. Philos Trans $R$ Soc Lond $B$ Biol Sci 304, 255-272, doi:10.1098/rstb.1984.0022 (1984).

49 Wong-Riley, M. \& Riley, D. A. The effect of impulse blockage on cytochrome oxidase activity in the cat visual system. Brain Res 261, 185-193, doi:10.1016/0006-8993(83)90622-4 (1983).

50 Storch, A., Ludolph, A. C. \& Schwarz, J. Dopamine transporter: involvement in selective dopaminergic neurotoxicity and degeneration. $J$ Neural Transm (Vienna) 111, 1267-1286, doi:10.1007/s00702-004-0203-2 (2004).

51 Boyden, E. S., Zhang, F., Bamberg, E., Nagel, G. \& Deisseroth, K. Millisecondtimescale, genetically targeted optical control of neural activity. Nat Neurosci 8 , 1263-1268, doi:10.1038/nn1525 (2005).

52 Yang, H. et al. Nucleus Accumbens Subnuclei Regulate Motivated Behavior via Direct Inhibition and Disinhibition of VTA Dopamine Subpopulations. Neuron 97, 434-449.e434, doi:10.1016/j.neuron.2017.12.022 (2018).

53 Marx, A., Backes, C., Meese, E., Lenhof, H. P. \& Keller, A. EDISON-WMW: Exact Dynamic Programing Solution of the Wilcoxon-Mann-Whitney Test. Genomics Proteomics Bioinformatics 14, 55-61, doi:10.1016/j.gpb.2015.11.004 (2016).

54 Berger, D. R., Seung, H. S. \& Lichtman, J. W. VAST (Volume Annotation and Segmentation Tool): Efficient Manual and Semi-Automatic Labeling of Large 3D Image Stacks. Front Neural Circuits 12, 88, doi:10.3389/fncir.2018.00088 (2018).

\section{Materials and Methods}

\section{Animals and AAV viruses}

DAT-CRE mice $\sim 15$ weeks used in this study were acquired from Xiaoxi Zhuang, The University of Chicago, and can also be found at Jackson Laboratory (https://www.jax.org/strain/020080). AAV-CAG-DIO-APEX2NES (Cyto-Apex) was acquired as a gift from the laboratory of Joshua Sanes (Harvard) and is now available at Addgene: \#79907. AAV-CAG-DIO-APEX2-MITO was generated in our lab by cloning the mitochondrial targeting sequencing from mito-V5-APEX2 (Addgene \#72480) and placing it on the 5' end of APEX2-NES in AAV-CAG-DIO-APEX2NES. Finally, the nuclear export sequence (NES) was removed from APEX2. AAV virus was generated at the University of North Carolina School of Medicine Vector Core facility (https://www.med.unc.edu/genetherapy/vectorcore/). Animal care, perfusion procedures, and AAV injections were followed according to animal regulations at the 
University of Chicago's Animal Resources Center (ARC) and approved IACUC protocols.

\section{$\underline{\text { AAV injections }}$}

AAV injections were performed using a standard stereotaxic frame. $70-100 \mathrm{nl}$ of virus $\left(\sim 2.9 \times 10^{12}\right.$ viral genomes $\left./ \mathrm{ml}\right)$ were bilaterally injected into the VTA using the stereotactic coordinates: 3.1 posterior of bregma, 0.55 lateral bregma, and 4.4 ventral of the dura. Mice were aged 4 weeks to allow for AAV expression before perfusion or cocaine sensitization experiments.

\section{Cocaine sensitization}

Mice were given a once daily intraperitoneal (IP) injection of either cocaine $(10 \mathrm{mg} / \mathrm{kg})$ or equivalent volume of saline every other day for a total of 4 injections. Immediately following each injection, mice were place in a novel environment where their locomotor activity was automatically monitored for one hour and then returned to their home cage.

\section{Apex2 staining and EM preparation.}

Brains were prepared in the same manner and as previously described ${ }^{22}$. Briefly, an anesthetized animal was first transcardially perfused with $10 \mathrm{ml} 0.1 \mathrm{M}$ Sodium Cacodylate (cacodylate) buffer, pH 7.4 (Electron microscopy sciences (EMS) followed by $20 \mathrm{ml}$ of fixative containing $2 \%$ paraformaldehyde (EMS), $2.5 \%$ glutaraldehyde (EMS) in $0.1 \mathrm{M}$ Sodium Cacodylate (cacodylate) buffer, pH 7.4 (EMS). The brain was removed and placed in fixative for at least 24 hours at $4^{\circ} \mathrm{C}$. A series of $300 \mu \mathrm{m}$ vibratome sections were prepared and put into fixative for 24 hours at $4^{\circ} \mathrm{C}$. Apex 2 precipitation and polymerization was performed by washing slices extensively in cacodylate buffer at room temperature, incubated in $50 \mathrm{mg} / \mathrm{ml} 3,3$ 'diaminobenzidine (DAB) for 1 hour at room temperature followed with $\mathrm{DAB} / 0.03 \%(\mathrm{v} / \mathrm{v}) \mathrm{H}_{2} \mathrm{O}_{2}$ until a visible precipitate forms (15-20 minutes). Slices were washed extensively in cacodylate buffer. Slices were reduced in $0.8 \%(\mathrm{w} / \mathrm{v})$ Sodium Hydrosulfite in $60 \%(\mathrm{v} / \mathrm{v}) 0.1 \mathrm{M}$ Sodium Bicarbonate $40 \%(\mathrm{v} / \mathrm{v}) 0.1 \mathrm{M}$ Sodium Carbonate buffer for 20 minutes and washed in cacodylate buffer. Brain slices that showed prominent Apex2 staining in the correct anatomical location were cut into smaller pieces surrounding the Apex2 positive tissue (i.e. NAc and VTA) and prepared for EM by staining sequentially with $2 \%$ osmium tetroxide (EMS) in cacodylate buffer, $2.5 \%$ potassium ferrocyanide (Sigma-Aldrich), thiocarbohydrazide, unbuffered $2 \%$ osmium tetroxide, $1 \%$ uranyl acetate, and $0.66 \%$ Aspartic acid buffered Lead (II) Nitrate with extensive rinses between each step with the exception of potassium ferrocyanide. The sections were then dehydrated in ethanol and propylene oxide and infiltrated with 812 Epon resin (EMS, Mixture: 49\% Embed 812, $28 \%$ DDSA, 21\% NMA, and 2.0\% DMP 30). The resin-infiltrated tissue was cured at $60^{\circ} \mathrm{C}$ for 3 days. Using a commercial ultramicrotome (Powertome, RMC), the cured block was trimmed to a $\sim 1.0 \mathrm{~mm} \times 1.5 \mathrm{~mm}$ rectangle and $\sim 2,000,40 \mathrm{~nm}$ thick sections were collected from each block on polyamide tape (Kapton) using an automated tape collecting device (ATUM, RMC) and assembled on silicon wafers as previously described ${ }^{18}$. The serial sections were acquired using backscattered electron detection 
with a Gemini 300 scanning electron microscope (Carl Zeiss), equipped with ATLAS software for automated wafer imaging. Dwell times for all datasets were 1.0 microsecond. For $20 \mathrm{~nm}$ and $6 \mathrm{~nm}$ resolution data sets, sections were brightness/contrast normalized and rigidly aligned using TrakEM2 (FIJI) followed by non-linear affine alignment using AlignTK on Argonne National Laboratory's super computer, Cooley. Different image processing tools were packaged into Python scripts that can be found here: https://github.com/Hanyu-Li/klab utils.

\section{Data Analysis}

Aligned datasets were manually skeletonized and annotated using the publicly available software, Knossos (https://knossos.app). Classes of cell types were identified by distinguishing anatomical properties: Apex2+ DA neurons (Somata and dendrites in the VTA and axons in NAc) were identified by their dark precipitate, Medium spiny neurons in the NAc by the presence of dendritic spines, and chemical synapse axons in the NAc by their formation of synapses on dendritic spines. Skeleton information was exported into tab delimited matrices using homemade Python scripts that compute skeleton features from the Knossos xml annotation file. Code is freely available here:

https://github.com/knorwood0/MNRVA. Quantification and plotting of different anatomical features were performed in Matlab and excel. Two-tailed Mann Whitney $U$ statistics test was used to test for significance ${ }^{53}$.

Mitochondria length

The length of mitochondria was measured in Knossos by measuring along the longest axis from one of the three orthogonal views $(x y, x z, y x)$. Dendrite diameter was measured by centering the mitochondria in the Knossos viewing window and then taking the average diameter between all three orthogonal axes.

Contact points

Contact points were found by tracing Apex2+ DA axons and marking in Knossos every point where an Apex2 negative neurite interdigitated with the DA axon. Interdigitation was determined by observing a neurite entering a DA axon and disappearing in the image stack using the three orthogonal views of Knossos. Neurites that passed over DA axons were not scored as contact points.

$3 \mathrm{D}$ rendering

3D manual segmentation was done using VAST ${ }^{54}$ and exported using MATLAB as .OBJ files which were then imported and rendered using Blender 2.79. 
Figure 1
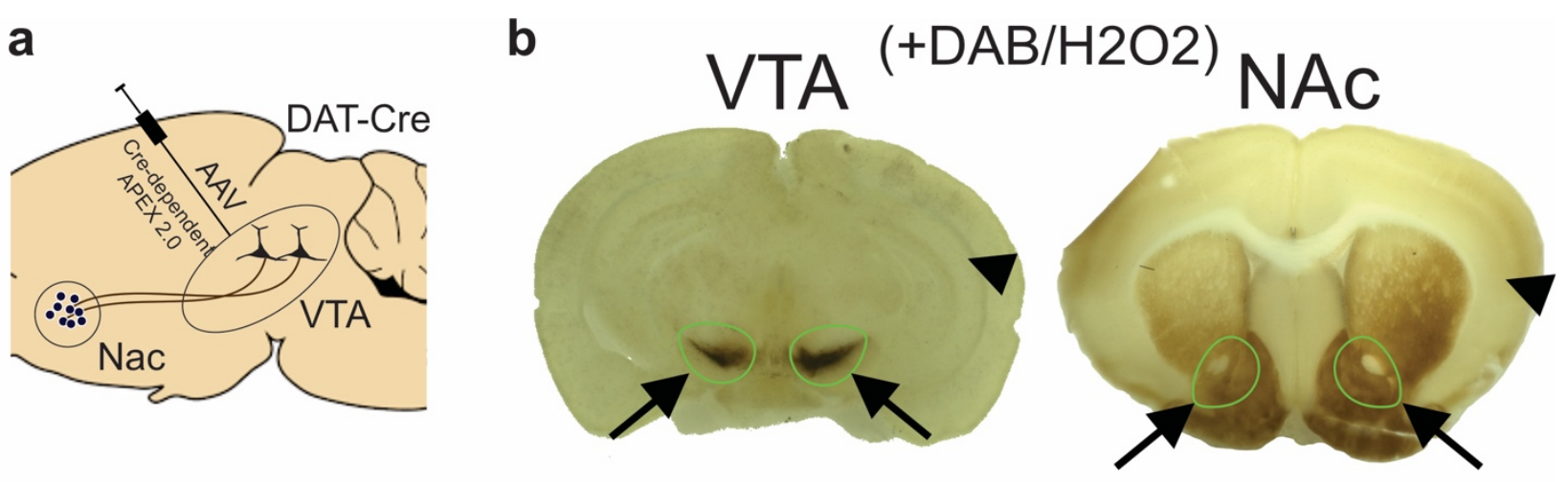

C
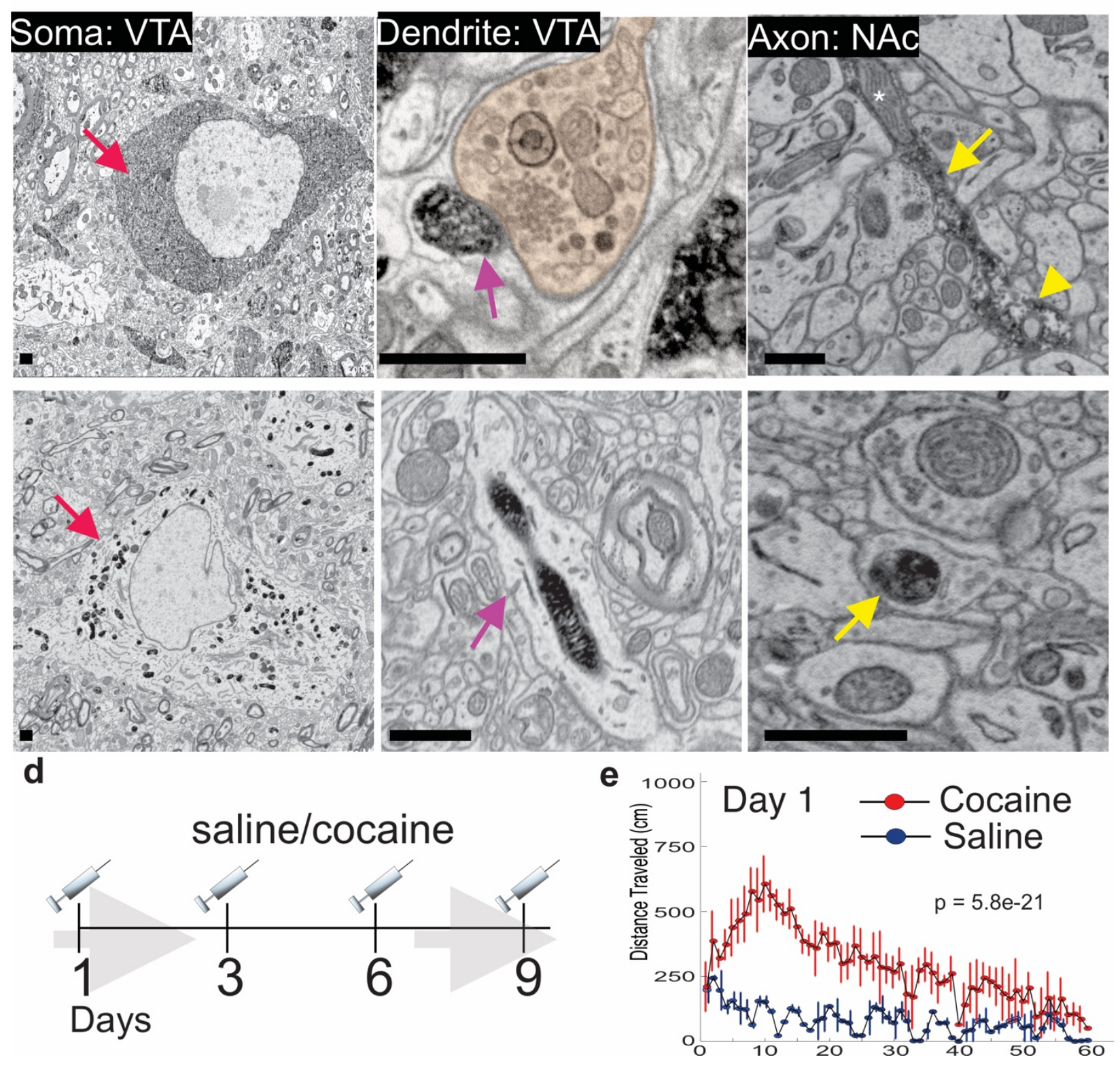
Figure 1. Experimental design for Dopamine connectomics. (a) AAVs expressing Cre-dependent Apex2 was biolaterally injected into the Ventral Tegmental Area (VTA) of transgenic mice expressing Cre in Dopamine Transporter positive neurons (DATCRE). (b) $\sim 4$ weeks after AAV injections, vibratome sections ( $\sim 300$ microns thick) show strong Apex2 labeling in VTA and NAc after staining with 3'3'-Diaminobenzidene (DAB) and Hydrogen Peroxide (H202) before EM processing (see Methods). Black arrows point to an Apex2 positive region and black arrowhead points to an Apex2 negative region. Green circles highlight the VTA and NAc region dissected out and processed for EM. (c) Representative EM images of cytoplasmic (top row) and mitochondrial (bottom row) Apex2 (Left panel: Apex2 Soma in the VTA (red arrows); Middle panel: Apex2 top panel shows a DA dendritic spine forming a synapse (purple arrow) with presynaptic bouton (orange) in the VTA, and the bottom panel shows a narrow DA dendrite expressing mitochondrial Apex; Right panel: Apex2 axon in the NAc with narrow (yellow arrow) and thick varicosities (yellow arrowhead). Cytosolic Apex2 does not fill up mitochondria (top panel, asterisk), and mitochondrial Apex2 (bottom) only fills up mitochondria. (d) Cartoon depicting cocaine sensitization protocol: Mice received a single intraperitoneal (IP) injection of cocaine $(10 \mathrm{mg} / \mathrm{kg})$ or saline, placed in a novel cage environment, and their locomotor activity was monitored for $1 \mathrm{hr}$. This procedure was repeated every 3 rd day for 4 rounds of injections. 4 days following the last injection (e.g. Day 13), mice were perfused and brains processed for Apex2/EM staining (see a and Methods). (e) Scatter plot of the average distance traveled $(\mathrm{cm})$ versus time for the first day of cocaine or saline injection $(n=3$ mice for each condition, error bars $=$ standard deviation of the mean, $P=5.8 \mathrm{e}-21)$. Scale bar $=1 \mu \mathrm{m}$. P-value: Mann-Whitney $\mathrm{U}$ test. 


\section{Figure 2}

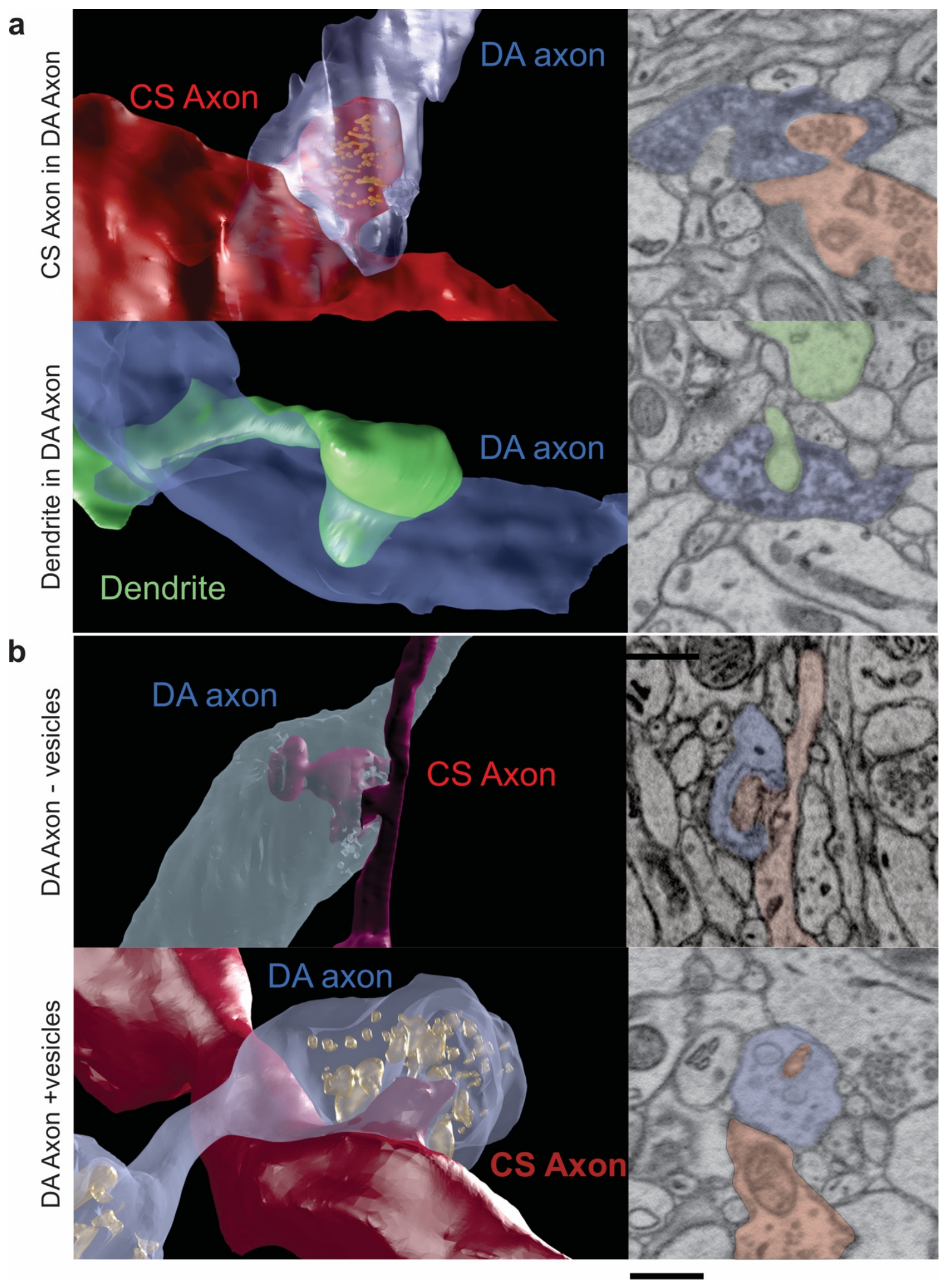


Figure 2. Dopamine contact points are physical interdigitations between DA axons and either NAc dendrites or afferent axons. (a) 3D renderings and matched 2D EM images of contact points between Apex+ DA axons (blue) and chemical synapse (CS) axons (red) (top panel) and Apex+ DA axons (blue) and dendrites (green) (bottom panel). (b) 3D renderings and matched EM image from a control mouse expressing mitochondrial Apex2 in DA neurons showing contact points between DA axons and CS axons where DA axon varicosities are either empty (top panel) or contain vesicles (bottom panel). Scale bar $=500 \mathrm{~nm}$. 
Figure 3

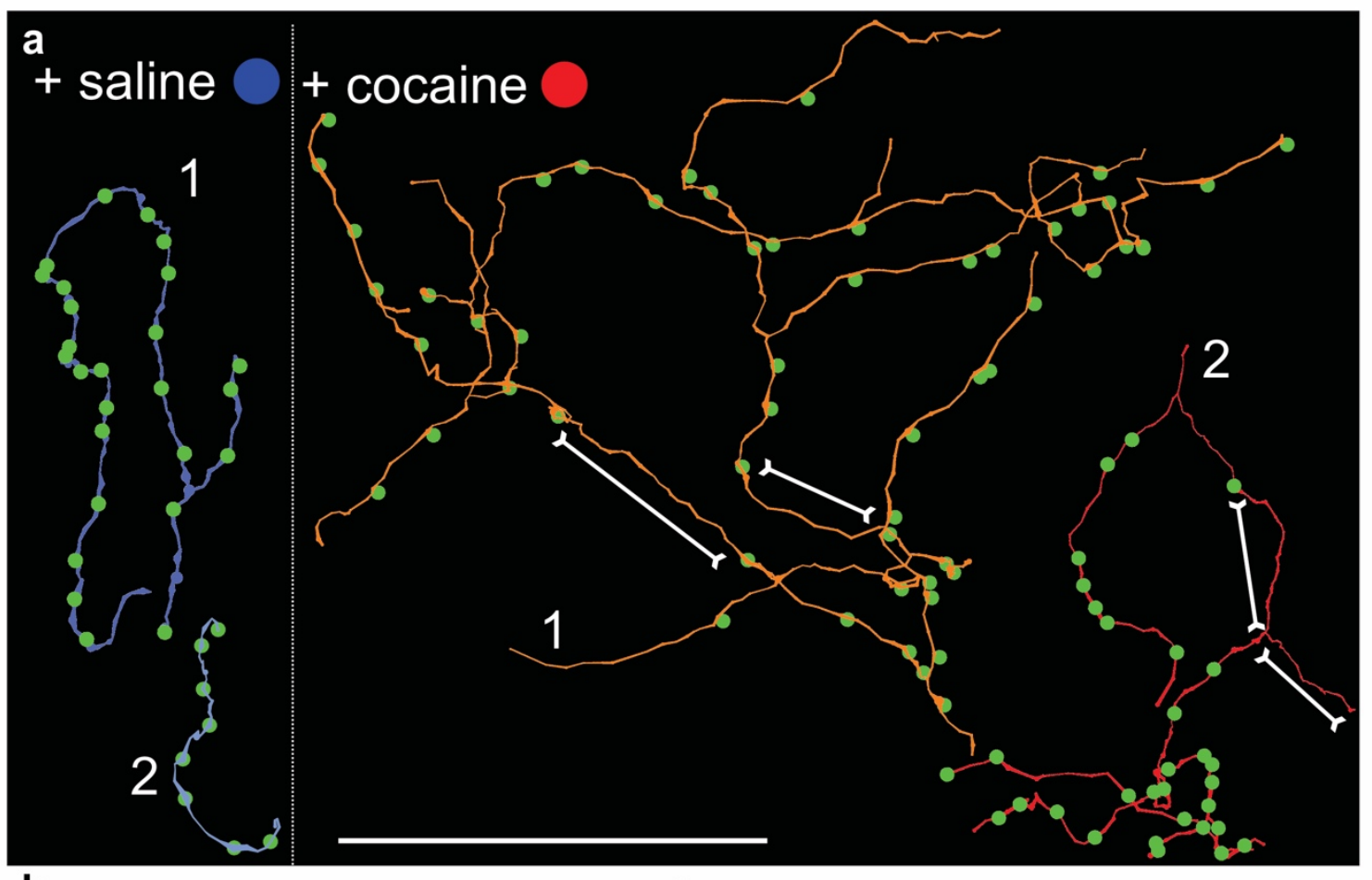

b
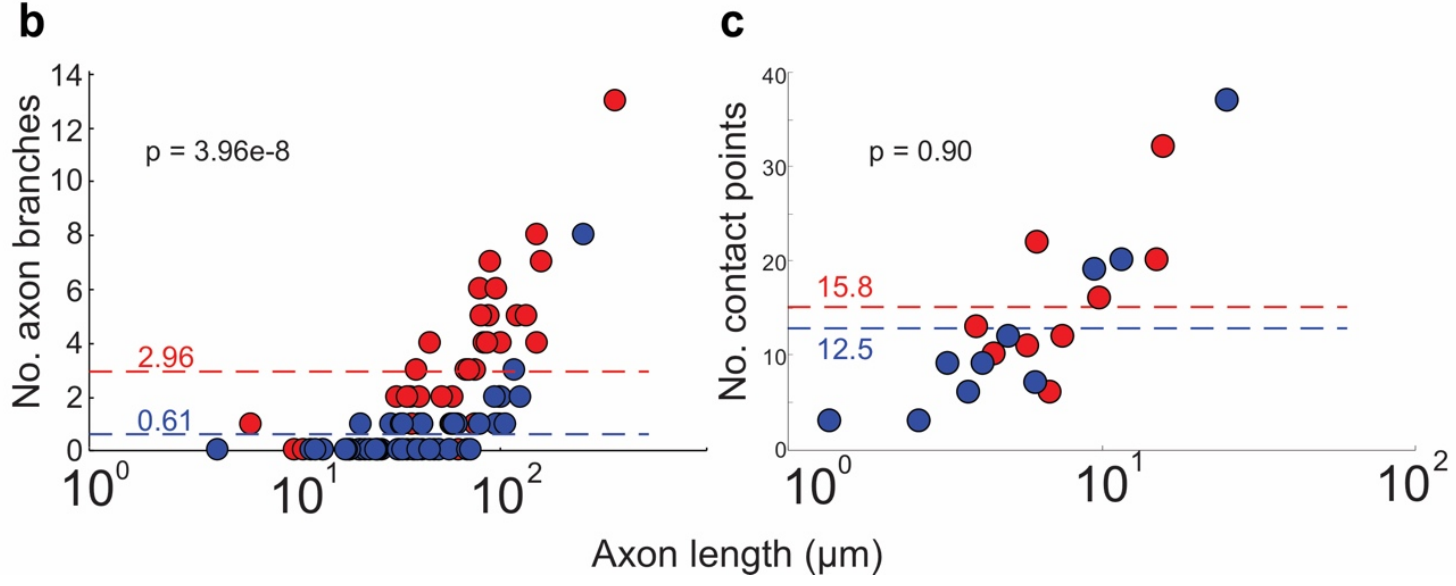
Figure 3. Cocaine increases branching of Apex2 dopamine axons in cocaine sensitized mice. (a) Two representative reconstructions of DA axons each from saline (left, blue) and cocaine (right, red) treated mice. Green circles represent contact points where other neurons interdigitate with the DA axon. (b) Scatter plot of the number of DA axons branches versus axon length $(\mu \mathrm{m})$ (+saline: $0.61 \pm 0.20$ branches/axon, $n=44$ axons; +cocaine: $2.96 \pm 0.43$ branches/axon, $n=41$ axons. $P=3.96 e-8$. (c) Scatter plot of the number of contact points versus total length of axon (+saline: $12.5 \pm 3.3$ contact points/axon, $\mathrm{n}=125$ contact points over 10 axons, $\mathrm{r} 2=0.94$; +cocaine: $15.8 \pm 2.6$ contact points/axon, $\mathrm{n}=142$ contact points over 9 axons, $\mathrm{r} 2=0.54$. $\mathrm{P}=0.9$ ). Data: mean \pm SEM. $P$-values: two-tailed Mann-Whitney $U$ test. 
Figure 4

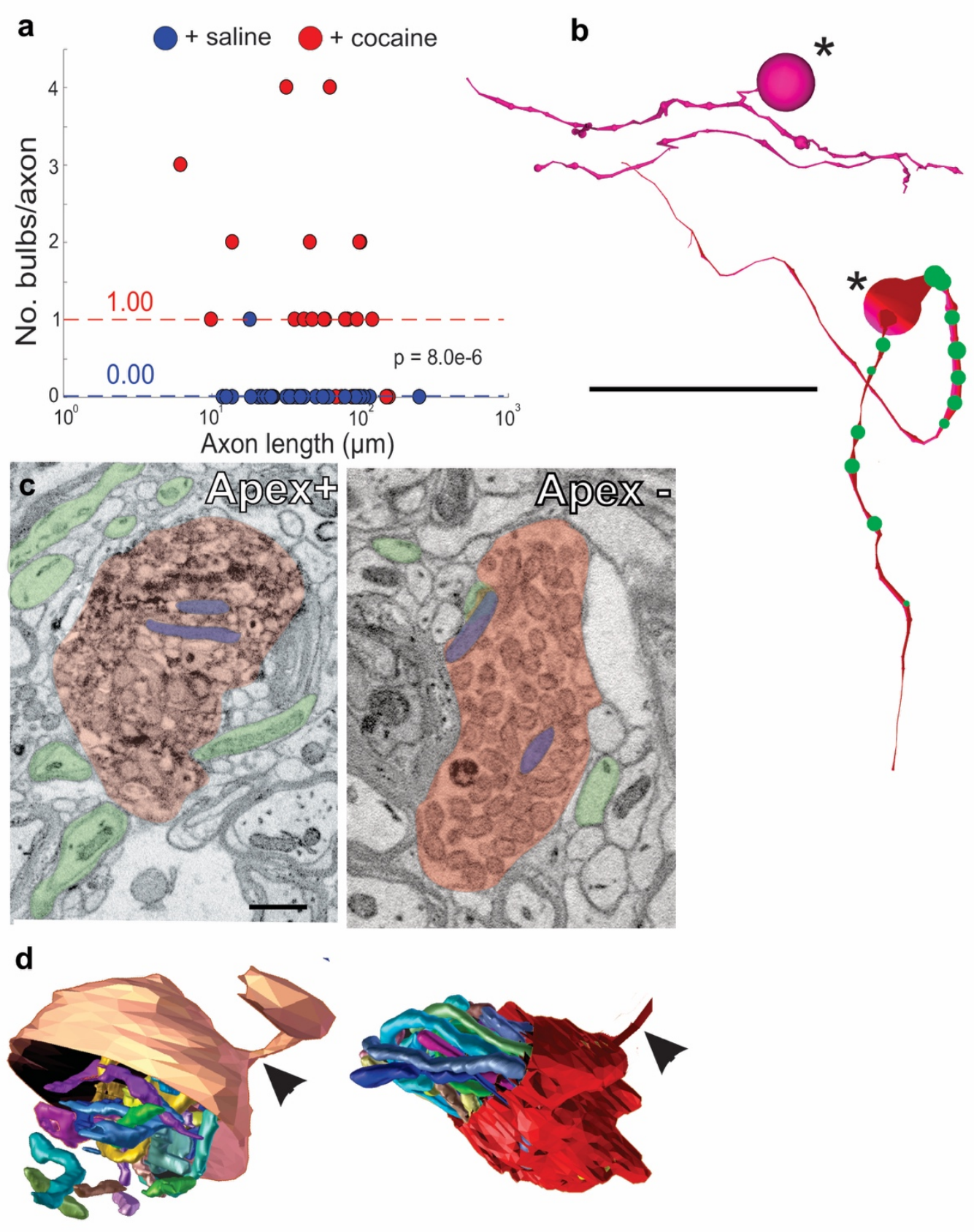


Figure 4. Cocaine results in the formation of large swellings filled with mitochondria in Apex2+ DA axons. (a) Scatter plot of the number of large swellings versus axon length $(\mu \mathrm{m})(+$ saline: $0.00 \pm 0.0$ swellings/axon, $n=29$ axons; + cocaine: $1.00 \pm 0.21$ swellings/axon, $n=30$ axons. $P=8.0 \mathrm{e}-6$. (b) Reconstructions of two representative Apex+ Dopamine axons with large swellings (asterix) and medium sized swellings (green spheres). Top reconstruction depicts an axon with a terminal axon bulb and bottom reconstruction shows one along the axon. (c) 2D EM images of Apex+ (top) and Apex- (bottom) large swellings (red) found in the NAC of cocaine treated animals. Both swellings are filled with mitochondria (examples highlighted in blue). Swellings are surrounded by local dendrites and axons (examples in green). (d) 3D segmentation of swellings from (b); swellings are filled with extremely long and coiled mitochondria and each swelling is attached to a thin axon (arrowheads). Scale bar: (B) $20 \mu \mathrm{m}$, (C) $500 \mathrm{~nm}$ Data: mean \pm SEM. $P$-values: two-tailed Mann-Whitney U test. 
Figure 5
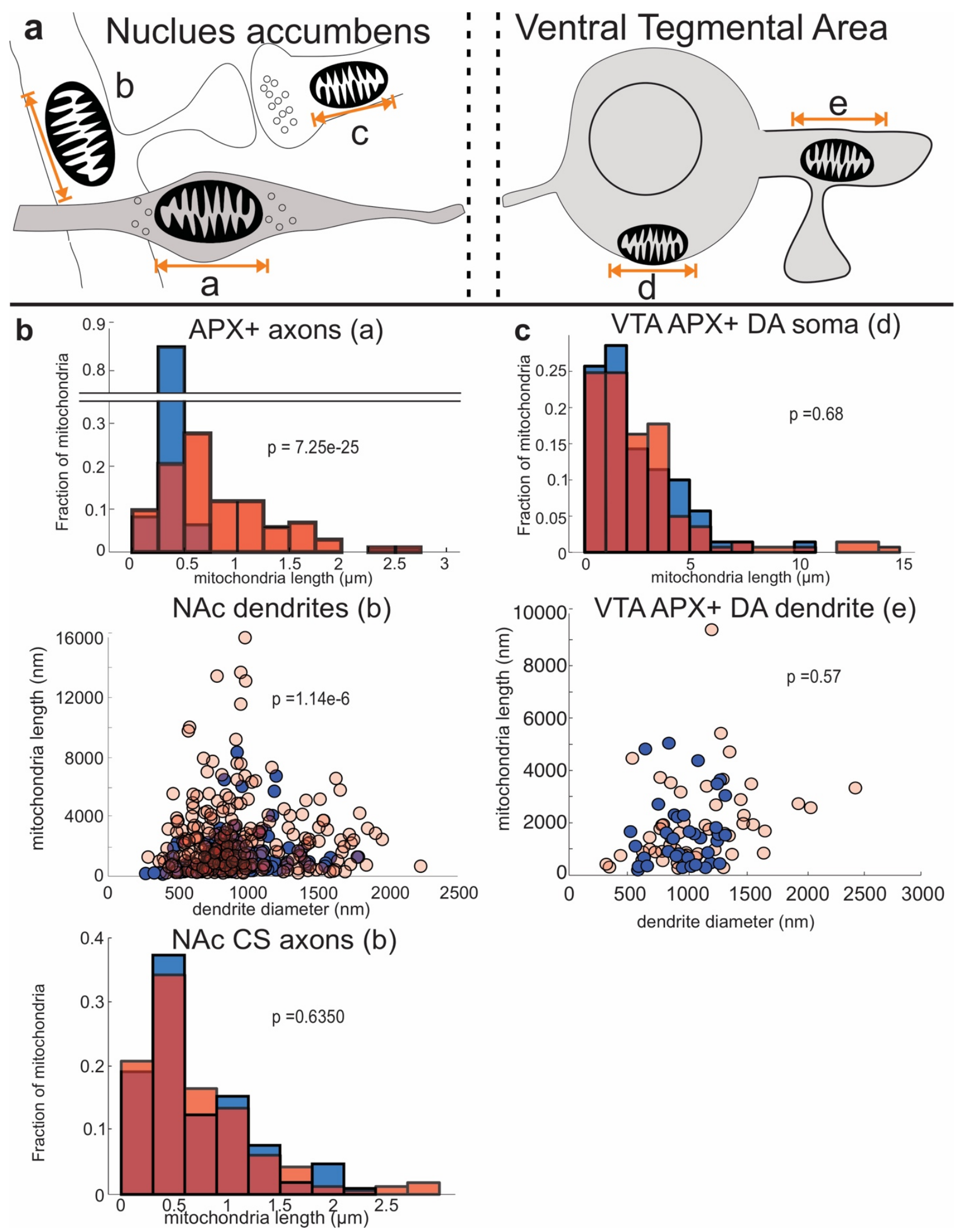
Figure 5. Cocaine results in increased mitochondria length in DA axons and MSN dendrites. (a) Cartoon depicting neurons where we measured mitochondrial length: Left: in the NAc, we measured mitochondria in: (a) Apex2+ DA axons, (b) MSN dendrites, and (c) excitatory axons making chemical synapses (CS axons). Right: in the VTA, we measured mitochondria in: (d) Apex2+ DA somata, and (e) Apex2+ DA dendrites. (b) Top: histogram of mitochondria length in Apex2+ DA axons (+saline: 0.36 $\pm 0.01 \mu \mathrm{m}, \mathrm{n}=162$ mitochondria across 42 axons; + cocaine: $0.79 \pm 0.05 \mu \mathrm{m}, \mathrm{n}=126$ mitochondria across 35 axons. $P=7.25 e-25$. Middle: scatter plot of mitochondria lengths versus dendrite diameter of NAc MSN dendrites (+ saline: $1.39 \pm 0.12$ mito length/dendrite diameter $(\mathrm{nm}), \mathrm{n}=132$ mitochondria across 50 dendrites; + cocaine: 3.0 $\pm 0.2 \mu \mathrm{m}, \mathrm{n}=260$ mitochondria across 41 dendrites. $\mathrm{P}=1.14 \mathrm{e}-6)$. Bottom: histogram of mitochondria lengths in NAc CS axons (+ saline: $0.70 \pm 0.05 \mu \mathrm{m}, \mathrm{n}=104$ mitochondria across 30 axons; + cocaine: $0.73 \pm 0.04 \mu \mathrm{m}, \mathrm{n}=164$ mitochondria across 57 axons. $\mathrm{P}=$ 0.64). (c) Top: Histogram of mitochondria length in Apex2+ DA Somata (+ saline: $2.46 \pm$ $0.24 \mu \mathrm{m}, \mathrm{n}=70$ mitochondria across 4 somata; + cocaine: $2.78 \pm 0.23 \mu \mathrm{m}, \mathrm{n}=141$ mitochondria across 5 somata. $P=0.68)$. Bottom: scatter plot of mitochondria lengths versus dendrite diameter of VTA Apex2+ DA dendrites (+saline: $1.74 \pm 0.25$ mito length/dendrite diameter $(\mathrm{nm}), \mathrm{n}=37$ mitochondria across 4 dendrites; + cocaine: 1.85 \pm 0.22 mito length/dendrite diameter $(\mathrm{nm}), \mathrm{n}=53$ mitochondria across 10 dendrites. $\mathrm{P}=$ 0.57. Data: mean \pm SEM. P-value: Mann-Whitney U test. 
Figures

a

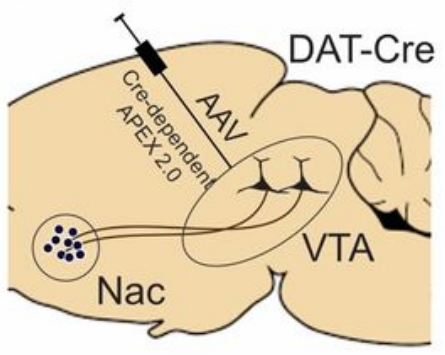

b

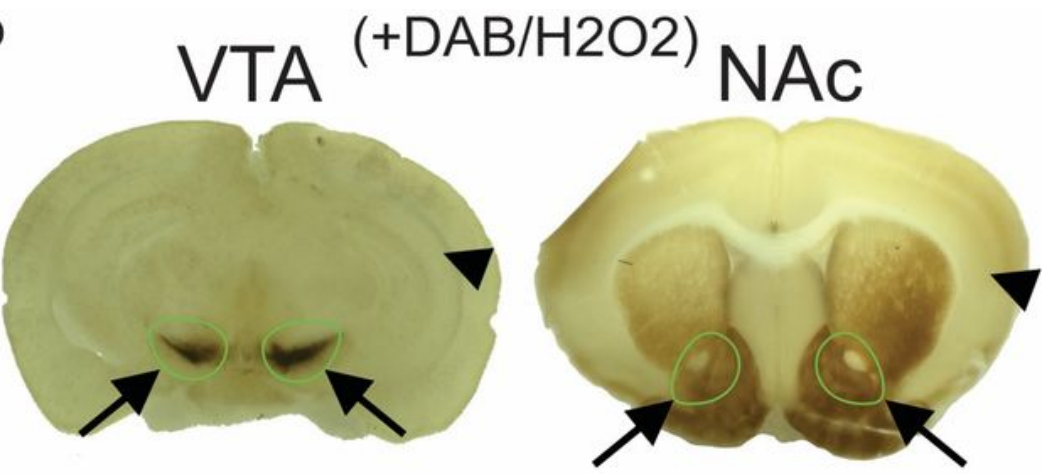

C

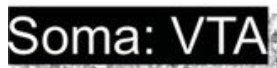

\section{A}

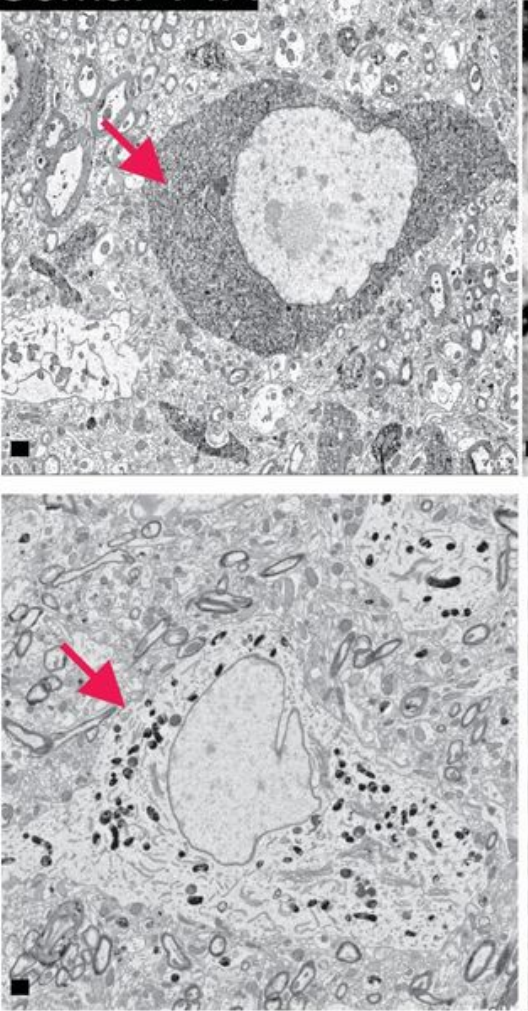

d

saline/cocaine

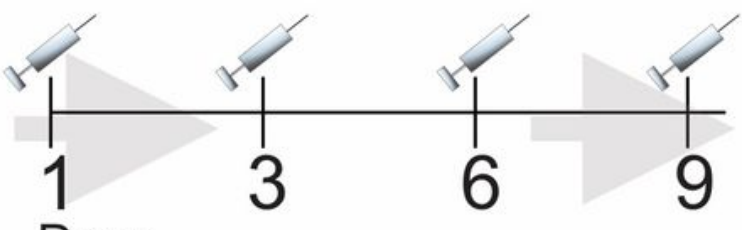

Days
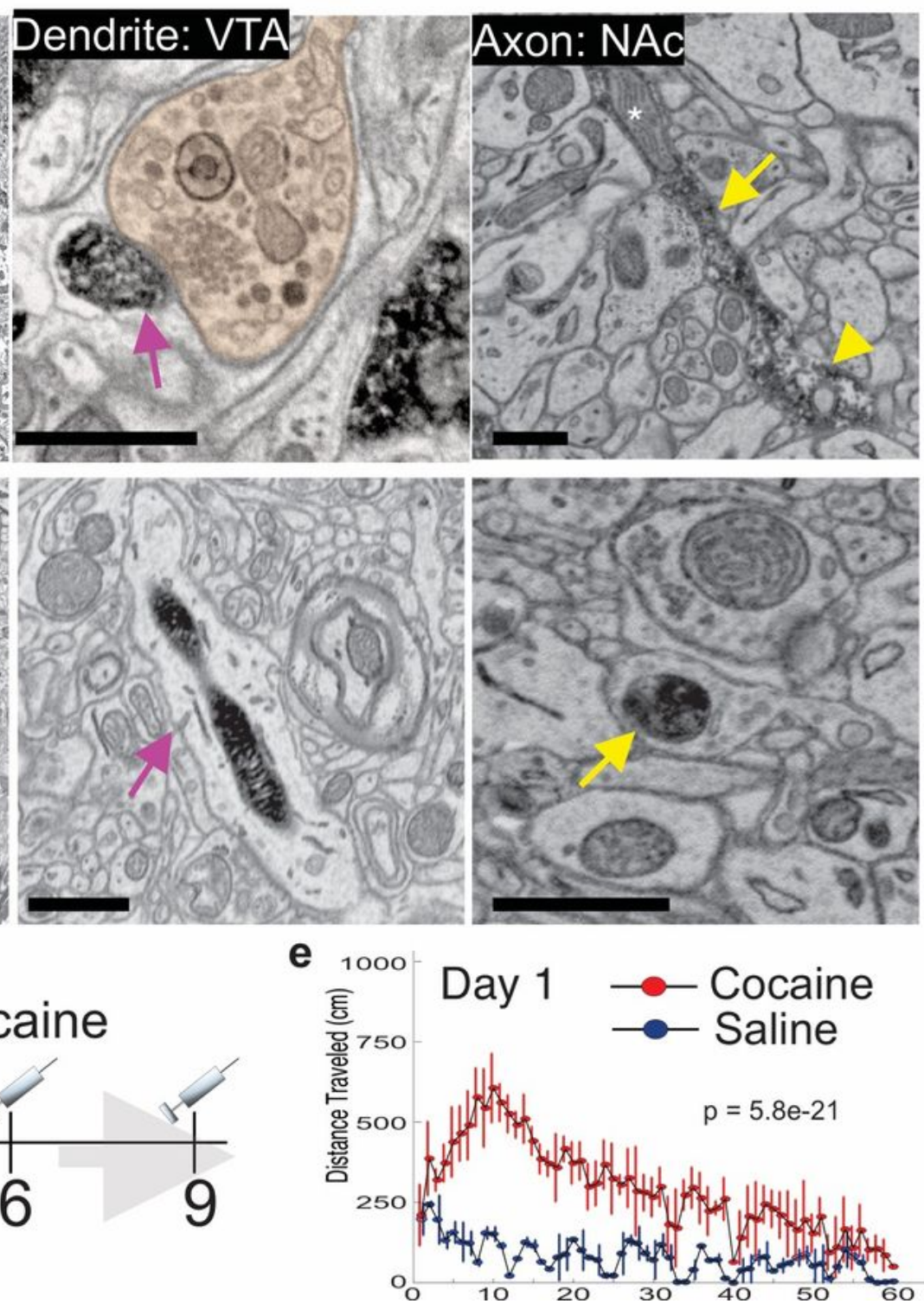

Figure 1

Experimental design for Dopamine connectomics. (a) AAVs expressing Cre-dependent Apex 2 was biolaterally injected into the Ventral Tegmental Area (VTA) of transgenic mice expressing Cre in Dopamine Transporter positive neurons (DATCRE). (b) 4 weeks after AAV injections, vibratome sections 
( 300 microns thick) show strong Apex2 labeling in VTA and NAc after staining with 3'3'Diaminobenzidene (DAB) and Hydrogen Peroxide (H202) before EM processing (see Methods). Black arrows point to an Apex 2 positive region and black arrowhead points to an Apex2 negative region. Green circles highlight the VTA and NAc region dissected out and processed for EM. (c) Representative EM images of cytoplasmic (top row) and mitochondrial (bottom row) Apex2 (Left panel: Apex2 Soma in the VTA (red arrows); Middle panel: Apex2 top panel shows a DA dendritic spine forming a synapse (purple arrow) with presynaptic bouton (orange) in the VTA, and the bottom panel shows a narrow DA dendrite expressing mitochondrial Apex; Right panel: Apex2 axon in the NAc with narrow (yellow arrow) and thick varicosities (yellow arrowhead). Cytosolic Apex2 does not fill up mitochondria (top panel, asterisk), and mitochondrial Apex2 (bottom) only fills up mitochondria. (d) Cartoon depicting cocaine sensitization protocol: Mice received a single intraperitoneal (IP) injection of cocaine $(10 \mathrm{mg} / \mathrm{kg})$ or saline, placed in a novel cage environment, and their locomotor activity was monitored for $1 \mathrm{hr}$. This procedure was repeated every 3rd day for 4 rounds of injections. 4 days following the last injection (e.g. Day 13), mice were perfused and brains processed for Apex2/EM staining (see a and Methods). (e) Scatter plot of the average distance traveled $(\mathrm{cm})$ versus time for the first day of cocaine or saline injection ( $n=3$ mice for each condition, error bars = standard deviation of the mean, $P=5.8 e-21)$. Scale bar $=1 \mu \mathrm{m}$. P-value: Mann-Whitney $\mathrm{U}$ test. 


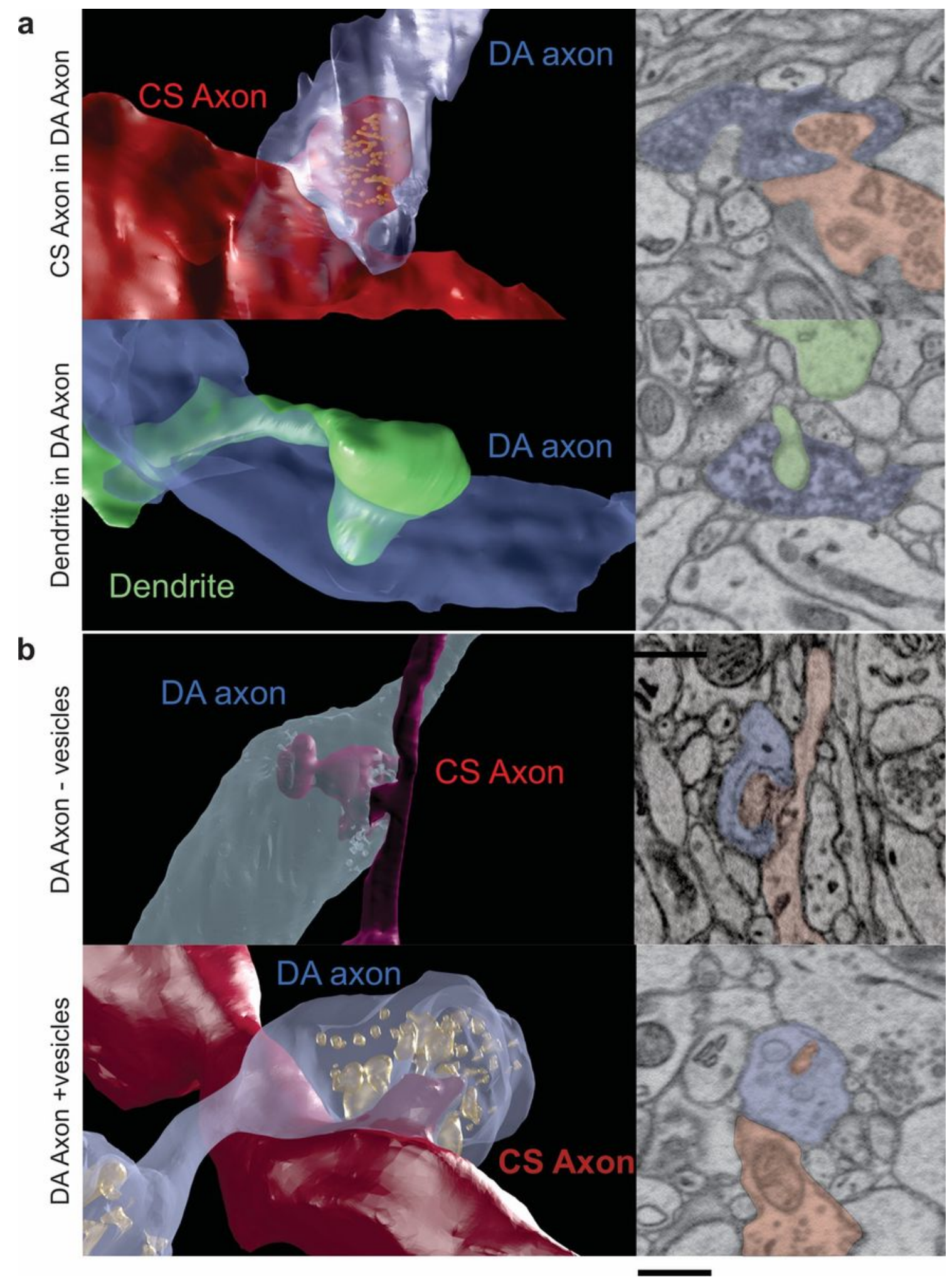

\section{Figure 2}

Dopamine contact points are physical interdigitations between DA axons and either NAc dendrites or afferent axons. (a) 3D renderings and matched 2D EM images of contact points between Apex+ DA axons (blue) and chemical synapse (CS) axons (red) (top panel) and Apex+ DA axons (blue) and dendrites (green) (bottom panel). (b) 3D renderings and matched EM image from a control mouse expressing mitochondrial Apex 2 in DA neurons showing contact points between DA axons and CS axons 
where DA axon varicosities are either empty (top panel) or contain vesicles (bottom panel). Scale bar = $500 \mathrm{~nm}$.

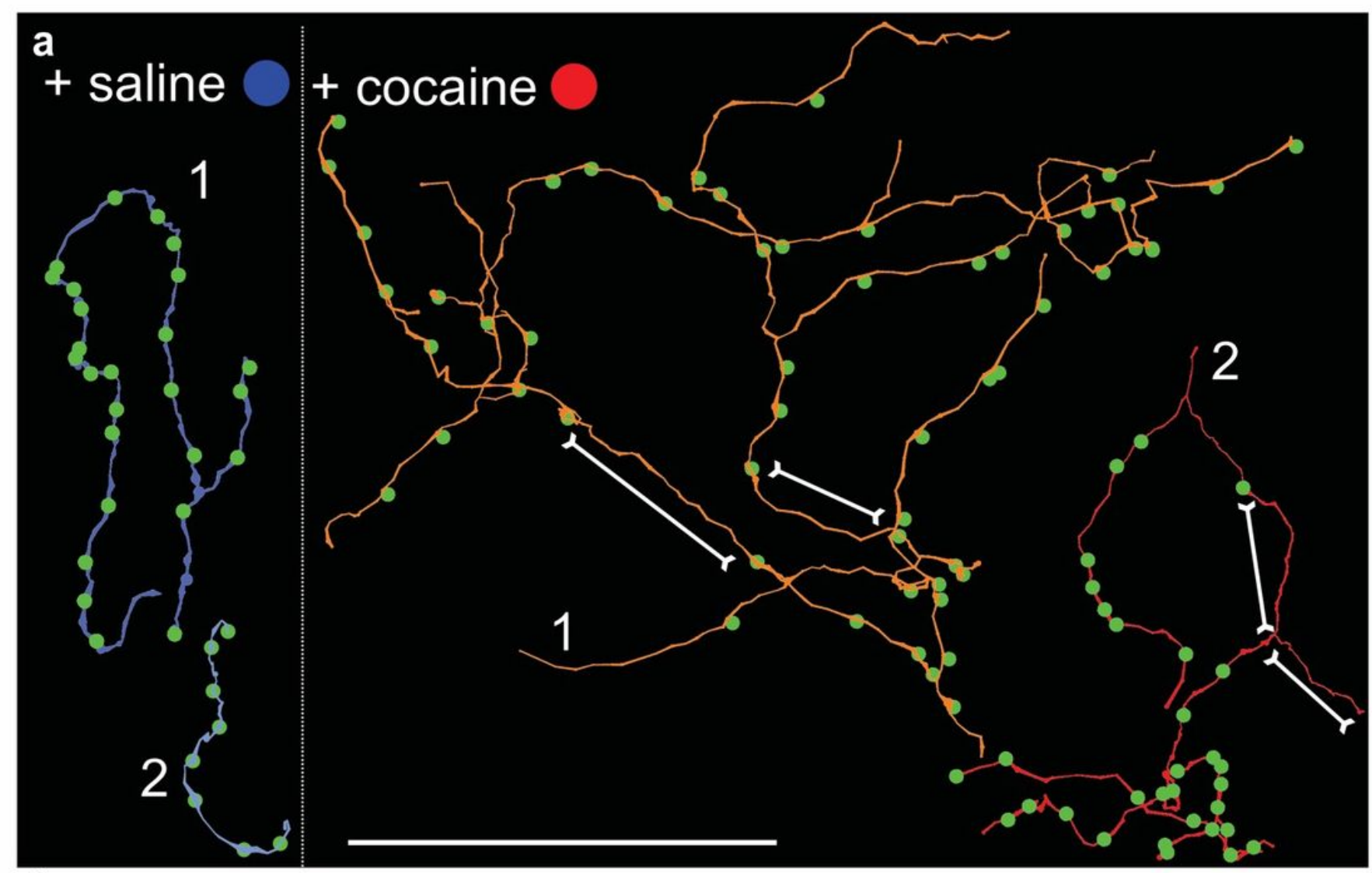

b

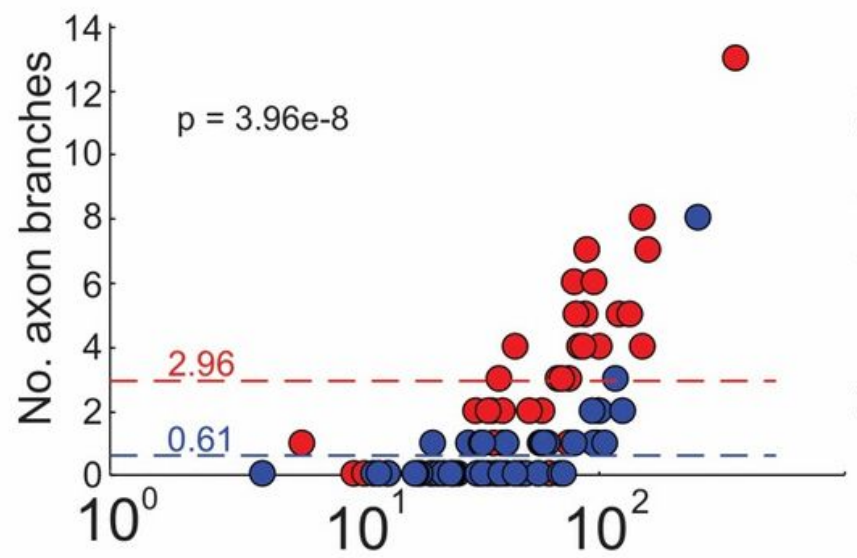

C

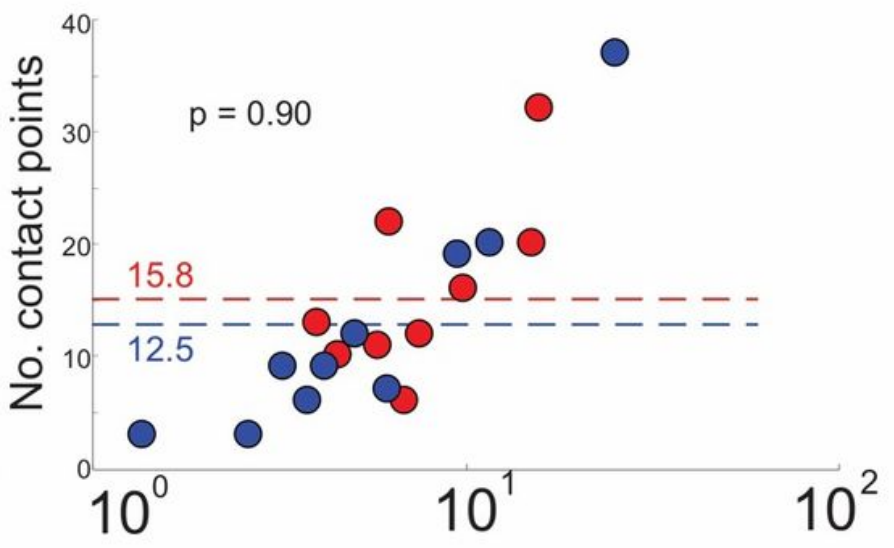

Axon length $(\mu \mathrm{m})$

\section{Figure 3}

Cocaine increases branching of Apex2 dopamine axons in cocaine sensitized mice. (a) Two representative reconstructions of DA axons each from saline (left, blue) and cocaine (right, red) treated mice. Green circles represent contact points where other neurons interdigitate with the DA axon. (b) Scatter plot of the number of DA axons branches versus axon length $(\mu \mathrm{m})$ (+saline: $0.61 \pm 0.20$ branches/axon, $n=44$ axons; +cocaine: $2.96 \pm 0.43$ branches/axon, $n=41$ axons. $P=3.96 \mathrm{e}-8$. (c) Scatter plot of the number of contact points versus total length of axon (+saline: $12.5 \pm 3.3$ contact points/axon, 
$\mathrm{n}=125$ contact points over 10 axons, $\mathrm{r} 2=0.94 ;$ +cocaine: $15.8 \pm 2.6$ contact points/axon, $\mathrm{n}=142$ contact points over 9 axons, $\mathrm{r} 2=0.54$. $\mathrm{P}=0.9$ ). Data: mean \pm SEM. P-values: two-tailed Mann-Whitney $U$ test.
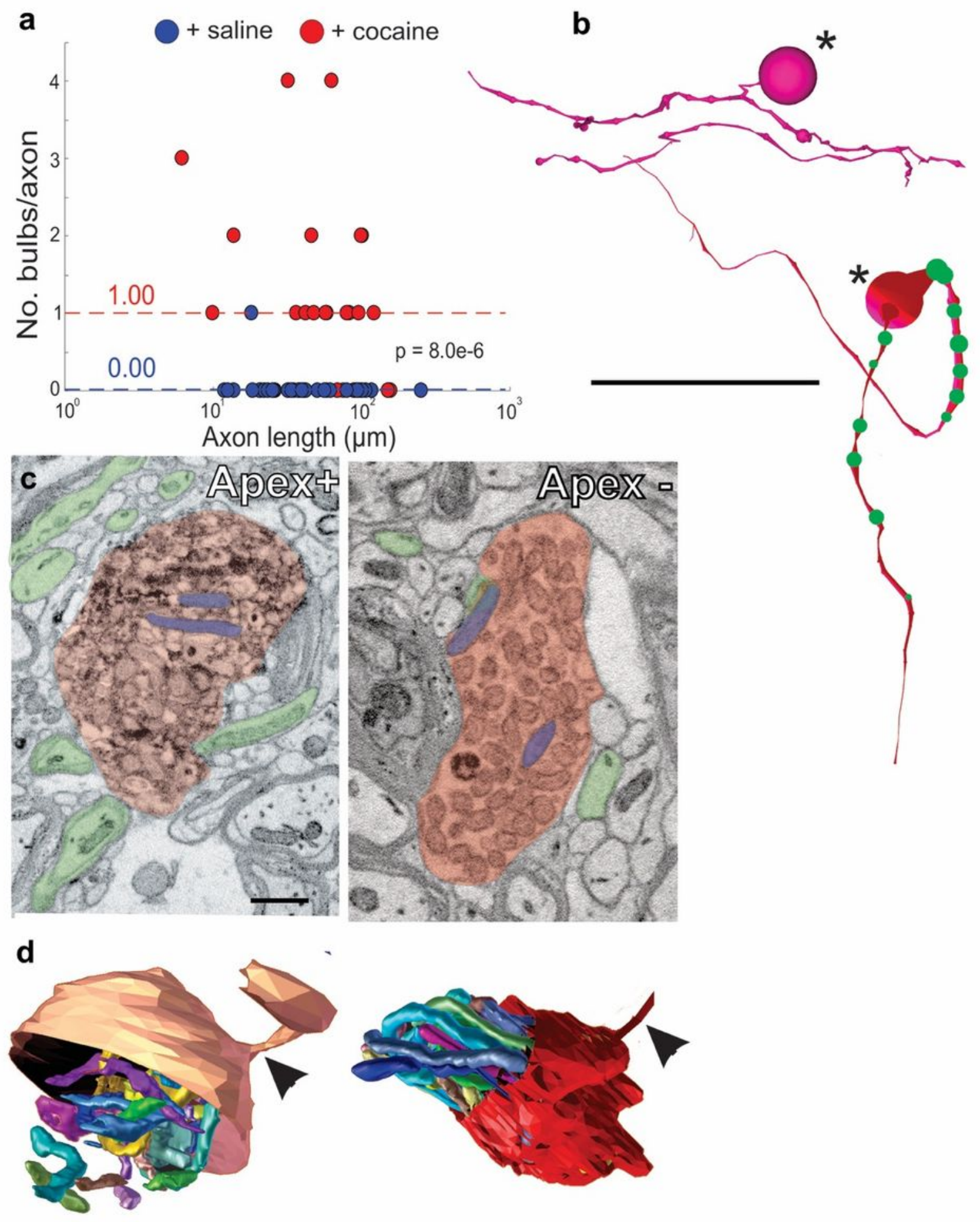

Figure 4

Cocaine results in the formation of large swellings filled with mitochondria in Apex2+ DA axons. (a) Scatter plot of the number of large swellings versus axon length $(\mu \mathrm{m})(+$ saline: $0.00 \pm 0.0$ 
swellings/axon, $n=29$ axons; + cocaine: $1.00 \pm 0.21$ swellings/axon, $n=30$ axons. $P=8.0 \mathrm{e}-6$. (b) Reconstructions of two representative Apex+ Dopamine axons with large swellings (asterix) and medium sized swellings (green spheres). Top reconstruction depicts an axon with a terminal axon bulb and bottom reconstruction shows one along the axon. (c) 2D EM images of Apex+ (top) and Apex- (bottom) large swellings (red) found in the NAC of cocaine treated animals. Both swellings are filled with mitochondria (examples highlighted in blue). Swellings are surrounded by local dendrites and axons (examples in green). (d) 3D segmentation of swellings from (b); swellings are filled with extremely long and coiled mitochondria and each swelling is attached to a thin axon (arrowheads). Scale bar: (B) $20 \mu \mathrm{m}$, (C) $500 \mathrm{~nm}$ Data: mean \pm SEM. P-values: two-tailed Mann-Whitney U test. 

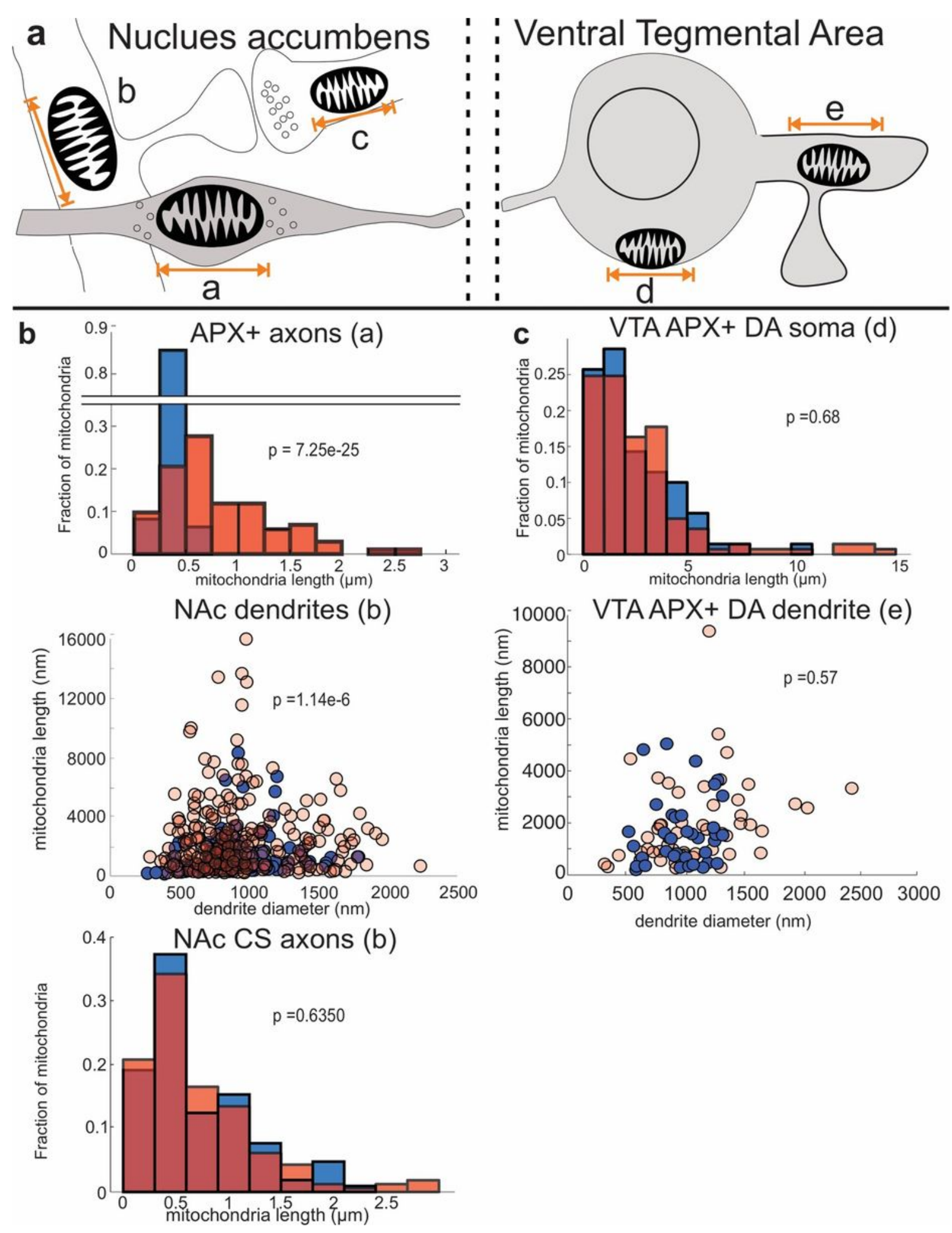

\section{Figure 5}

Cocaine results in increased mitochondria length in DA axons and MSN dendrites. (a) Cartoon depicting neurons where we measured mitochondrial length: Left: in the NAc, we measured mitochondria in: (a) Apex2+ DA axons, (b) MSN dendrites, and (c) excitatory axons making chemical synapses (CS axons). Right: in the VTA, we measured mitochondria in: (d) Apex2+ DA somata, and (e) Apex2+ DA dendrites. (b) Top: histogram of mitochondria length in Apex2+ DA axons (+saline: $0.36 \pm 0.01 \mu \mathrm{m}, \mathrm{n}=162$ 
mitochondria across 42 axons; + cocaine: $0.79 \pm 0.05 \mu \mathrm{m}, \mathrm{n}=126$ mitochondria across 35 axons. $\mathrm{P}=$ $7.25 \mathrm{e}-25$. Middle: scatter plot of mitochondria lengths versus dendrite diameter of NAc MSN dendrites (+ saline: $1.39 \pm 0.12$ mito length/dendrite diameter $(\mathrm{nm}), \mathrm{n}=132$ mitochondria across 50 dendrites; + cocaine: $3.0 \pm 0.2 \mu \mathrm{m}, \mathrm{n}=260$ mitochondria across 41 dendrites. $P=1.14 \mathrm{e}-6)$. Bottom: histogram of mitochondria lengths in NAc CS axons (+ saline: $0.70 \pm 0.05 \mu \mathrm{m}, \mathrm{n}=104$ mitochondria across 30 axons; + cocaine: $0.73 \pm 0.04 \mu \mathrm{m}, \mathrm{n}=164$ mitochondria across 57 axons. $P=0.64)$. (c) Top: Histogram of mitochondria length in Apex2+ DA Somata (+ saline: $2.46 \pm 0.24 \mu \mathrm{m}, \mathrm{n}=70$ mitochondria across 4 somata; + cocaine: $2.78 \pm 0.23 \mu \mathrm{m}, \mathrm{n}=141$ mitochondria across 5 somata. $\mathrm{P}=0.68)$. Bottom: scatter plot of mitochondria lengths versus dendrite diameter of VTA Apex2+DA dendrites (+saline: $1.74 \pm 0.25$ mito length/dendrite diameter $(\mathrm{nm}), \mathrm{n}=37$ mitochondria across 4 dendrites; + cocaine: $1.85 \pm 0.22$ mito length/dendrite diameter $(n m), n=53$ mitochondria across 10 dendrites. $P=0.57$. Data: mean $\pm S E M$. $P$ value: Mann-Whitney U test.

\section{Supplementary Files}

This is a list of supplementary files associated with this preprint. Click to download.

- Dopaminesupplementaryfinalnatcomms.docx

- Kasthurinrreportingsummary.pdf 